\title{
REVIEW ARTICLE OPEN \\ Stem cell-based bone and dental regeneration: a view of microenvironmental modulation
}

\author{
Chenxi Zheng ${ }^{1}$, Ji Chen ${ }^{1}$, Shiyu Liu $^{1}$ and Yan Jin (iD ${ }^{1}$
}

In modern medicine, bone and dental loss and defects are common and widespread morbidities, for which regenerative therapy has shown great promise. Mesenchymal stem cells, obtained from various sources and playing an essential role in organ development and postnatal repair, have exhibited enormous potential for regenerating bone and dental tissue. Currently, mesenchymal stem cells (MSCs)-based bone and dental regeneration mainly includes two strategies: the rescue or mobilization of endogenous MSCs and the application of exogenous MSCs in cytotherapy or tissue engineering. Nevertheless, the efficacy of MSCbased regeneration is not always fulfilled, especially in diseased microenvironments. Specifically, the diseased microenvironment not only impairs the regenerative potential of resident MSCs but also controls the therapeutic efficacy of exogenous MSCs, both as donors and recipients. Accordingly, approaches targeting a diseased microenvironment have been established, including improving the diseased niche to restore endogenous MSCs, enhancing MSC resistance to a diseased microenvironment and renormalizing the microenvironment to guarantee MSC-mediated therapies. Moreover, the application of extracellular vesicles (EVs) as cell-free therapy has emerged as a promising therapeutic strategy. In this review, we summarize current knowledge regarding the tactics of MSC-based bone and dental regeneration and the decisive role of the microenvironment, emphasizing the therapeutic potential of microenvironment-targeting strategies in bone and dental regenerative medicine.

International Journal of Oral Science (2019)11:23; https://doi.org/10.1038/s41368-019-0060-3

\section{INTRODUCTION}

Bone and dental loss and defects caused by diseases and trauma have become a global concern with high incidence, which seriously affects the health and life quality of the whole population and lays a heavy financial burden on society. ${ }^{1,2}$ Currently, autogenous bone transplantation is the gold standard treatment for bone defects. ${ }^{3,4}$ For oral diseases, dental prostheses, periodontal treatment and dental implants remain the mainstream therapies. ${ }^{2,5}$ However, the application of autogenous bone transplantation is seriously restrained by limitations of sources, difficulty in graft harvest and morbidity of donor site. ${ }^{6,7}$ Moreover, present therapies for oral diseases can only improve clinical diagnostic parameters and halt disease progression but fail to regenerate lost tissue., ${ }^{2,8}$ Therefore, new technologies are in high demand to achieve excellent regeneration of bone and dental tissues.

Mesenchymal stem cells (MSCs), which can be isolated from different tissues and possess self-renewal and multiple differentiation potential, play an essential role in organ development and postnatal repair. ${ }^{9-11}$ A variety of studies, via animal models and clinical trials, have demonstrated that both endogenous and exogenous MSCs hold enormous promise in regenerative medicine for bone and tooth, ${ }^{12-15}$ among which bone marrow MSCs (BMMSCs) have received much attention. In addition, adipose-derived MSCs (ADMSCs) ${ }^{16,17}$ and dental stem cells (DSCs), ${ }^{18,19}$ including dental pulp stem cells (DPSCs), periodontal ligament stem cells (PDLSCs), stem cells from human exfoliated deciduous teeth (SHED), stem cells from the apical papilla (SCAP) and dental follicle cells (DFCs), have emerged as attractive cell sources for bone and dental regeneration due to their ease of accessibility and relative abundance. In addition to differentiation potential, the ability of MSCs to regulate the function of other cells and to modulate the systemic inflammatory condition via cell-cell interaction or paracrine mechanism also contributes to their therapeutic efficacy. ${ }^{20,21}$ Presently, there are two main strategies of MSC-based bone and dental regeneration: the rescue or mobilization of endogenous MSCs and the application of exogenous MSCs in cytotherapy or tissue engineering. Nevertheless, despite much progress, establishing safe, effective and simple stem cell-based approaches for bone and dental repair and regeneration remains a tremendous challenge, ${ }^{8,14,15,22}$ especially considering the adverse effects of a diseased microenvironment. ${ }^{21}$

In recent years, the microenvironment has been uncovered to exert enormous influence on the physical functions and pathologic changes as well as the therapeutic effects of stem cells. ${ }^{23-25}$ Physiologically, the niche where MSCs reside consists of a variety of tissue components, cell populations and soluble factors, which tightly regulate the behaviours of MSCs. ${ }^{26-28}$ Under pathological conditions, such as osteoporosis and periodontitis, both the viability and differentiation of MSCs are seriously impaired, leading to disease aggravation and impaired tissue healing. ${ }^{21,29-31}$ Furthermore, in cytotherapy and tissue engineering, the microenvironments of donors and recipients play a pivotal role in determining the regenerative efficacy of transplanted $\mathrm{MSCs}$, 32,33

\footnotetext{
${ }^{1}$ State Key Laboratory of Military Stomatology \& National Clinical Research Center for Oral Diseases \& Shaanxi International Joint Research Center for Oral Diseases, Center for Tissue Engineering, School of Stomatology, The Fourth Military Medical University, Xi'an, Shaanxi, China Correspondence: Shiyu Liu (liushiyu@vip.163.com) or Yan Jin (yanjin@fmmu.edu.cn)
}

Received: 28 January 2019 Revised: 28 May 2019 Accepted: 12 June 2019

Published online: 19 August 2019 
further indicating a critical role of cell-microenvironment interactions in MSC-mediated bone and dental regeneration.

In addition to accumulating evidence identifying the prominence of the microenvironment in MSC-based regenerative therapies, solutions have been developed, such as the improvement of the microenvironment to restore endogenous MSC function, the enhancement of MSC resistance to a diseased microenvironment, and the restoration of the recipient microenvironment to benefit transplanted MSCs. Notably, after being transplanted into recipients, MSCs act as potent microenvironment modulators in both tissue engineering and cytotherapy. ${ }^{34-38}$ Furthermore, cell-free therapies represented by the application of MSC-derived extracellular vesicles (EVs) have become a promising alternative to whole-cell treatment. ${ }^{39-41}$ In this review, we overview the principles and cutting-edge progress of MSC-based tactics in bone and tooth regeneration and further highlight the involvement of the microenvironment, especially under pathological conditions. We also propose strategies to optimize MSC-based regeneration of bone and dental tissues, mainly focusing on modulating the microenvironment.

\section{MSCS IN BONE AND TOOTH REGENERATION}

Among the various stem cell types used for cytotherapy and tissue engineering, MSCs are currently proposed as an attractive cell source for bone and tooth regeneration due to their potential for differentiation into osteoblasts or odontoblasts, ability to modulate systematic immunity, and lack of ethical controversies. ${ }^{12,14,15,22}$ In addition to being classically obtained from bone marrow, MSCs can also be isolated from diverse neonatal and adult tissues, which provide more accessible sources of MSCs for bone and dental tissue regenerative therapies. ${ }^{18,42,43}$

\section{BMMSCs}

BMMSCs were first discovered by Friedenstein et al. ${ }^{44}$ as a subpopulation of non-haematopoietic stromal cells residing in bone marrow that were able to self-renew and differentiate into multiple cell types. Since then, BMMSCs have become the most extensively studied MSCs for bone regeneration due to their intimate involvement in bone physiology and pathology.,45 During adult life, bone homoeostasis maintenance depends largely on the balance between bone formation and resorption, ${ }^{46,47}$ which, at the cellular level, is intensely modulated by BMMSCs via differentiating into osteoblasts and regulating osteoclasts' activities. $^{10,48}$ Pathologically, BMMSC dysfunction has been revealed to be a critical cellular mechanism underlying various bone disorders, especially osteoporosis. ${ }^{21,30,31}$ More importantly, BMMSCs act as potent microenvironmental modulators that exert enormous anti-inflammatory effects after systemic transplantation, which benefit diverse tissues/organs, including bone. $^{35,36,41}$ Accordingly, a variety of studies have shown promising therapeutic potential of BMMSCs in osteopenia and bone defects via cytotherapy or tissue engineering construction. $33,41,49-52$

\section{ADMSCs}

Since their discovery by Zuk et al. ${ }^{53}$ in 2001, ADMSCs have been increasingly demonstrated to hold great promises in regenerative medicine. Similar to BMMSCs, ADMSCs display steady growth kinetics in vitro and are able to differentiate into various cell types, including osteocytes, chondrocytes and adipocytes. ${ }^{16,17}$ In addition, with a prevalence of lipoaspirates and less morbidity to the host during procurement, ADMSCs are, to some extent, more advantageous than BMMSCs due to easy accessibility and abundant supply. ${ }^{16,17,54}$ Furthermore, compared to BMMSCs that are prone to pathological factors of bone, ADMSCs demonstrate functional maintenance in various bone pathological conditions, as demonstrated by the preservation of cell viability,

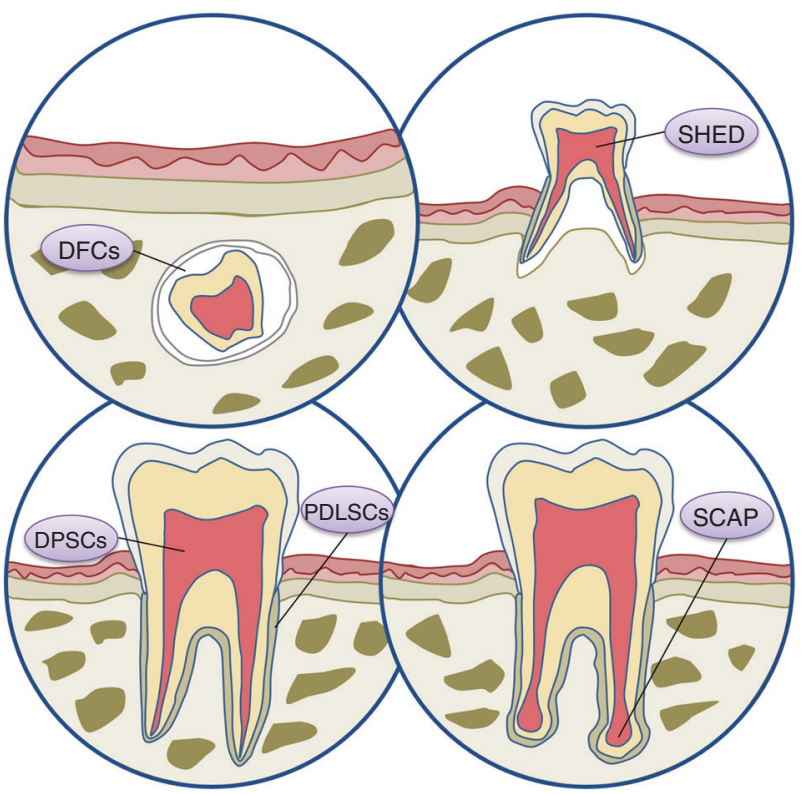

Fig. 1 Stem cell populations derived from different dental tissues/ regions that constitute appealing MSC sources for bone and dental regeneration. DFCs, stem cells from dental follicle; SHED, stem cells from human exfoliated deciduous teeth; DPSCs, stem cells from dental pulp; PDLSCs, stem cells from PDL; SCAP, stem cells from the apical papilla

differentiation capacity, ${ }^{29,55-58}$ and more importantly, therapeutic efficacy. ${ }^{59-61}$ Recent studies have revealed the efficacy of ADMSCs in repairing critical-sized bone defects, improving osteopenia and constructing engineered bone grafts and have proposed these cells as an excellent alternative to BMMSCs. ${ }^{59-63}$

\section{DSCs}

A variety of stem cell populations have been obtained from diverse parts of the tooth with a common neural crest origin and generic MSC-like properties, including the expression of specific surface markers and potential to differentiate into mesenchymal cell lineages ${ }^{18,19}$ (Fig. 1). Remarkably, DSCs exhibit many advantages, such as easy accessibility, abundant source and less inconvenience to donors, ${ }^{14,54}$ which constitutes an appealing source of autologous MSCs, especially for the regeneration of pulp tissue and periodontal ligament (PDL) and the production of partial or whole tooth structures for biological implant construction. $^{14,18,19,54}$

DPSCs. Derived from pulp that is entrapped within the pulp chamber and possessing good reparative and regenerative abilities, DPSCs are the first DSCs to be discovered by Gronthos et al. $^{64}$ in 2000 with self-renewal capability and multilineage differentiation potential. DPSCs are essential for postnatal tooth homoeostasis and repair due to their ability to replenish odontoblasts during the restoration of dentin. ${ }^{65-67}$ In addition, since DPSCs are of neural origin, these cells are able to differentiate into functionally active neurons and glial cells upon proper environmental stimulation. ${ }^{68,69}$ In addition, DPSCs demonstrate a distinguishing capacity to secrete neurotrophic factors that contribute to neuroprotection and neurite outgrowth. ${ }^{68,69}$ More importantly, recent studies have revealed that DPSCs reside in a neurovascular bundle niche. ${ }^{70}$ Intriguingly, in accord with their perivascular residence, DPSCs possess strong angiogenic ability, ${ }^{69}$ as shown by their abilities to secrete an array of angiogenic regulatory factors and to generate capillary-like structures under particular environmental conditions. Taken together, the MSC-like and neurovascular properties of DPSCs, 


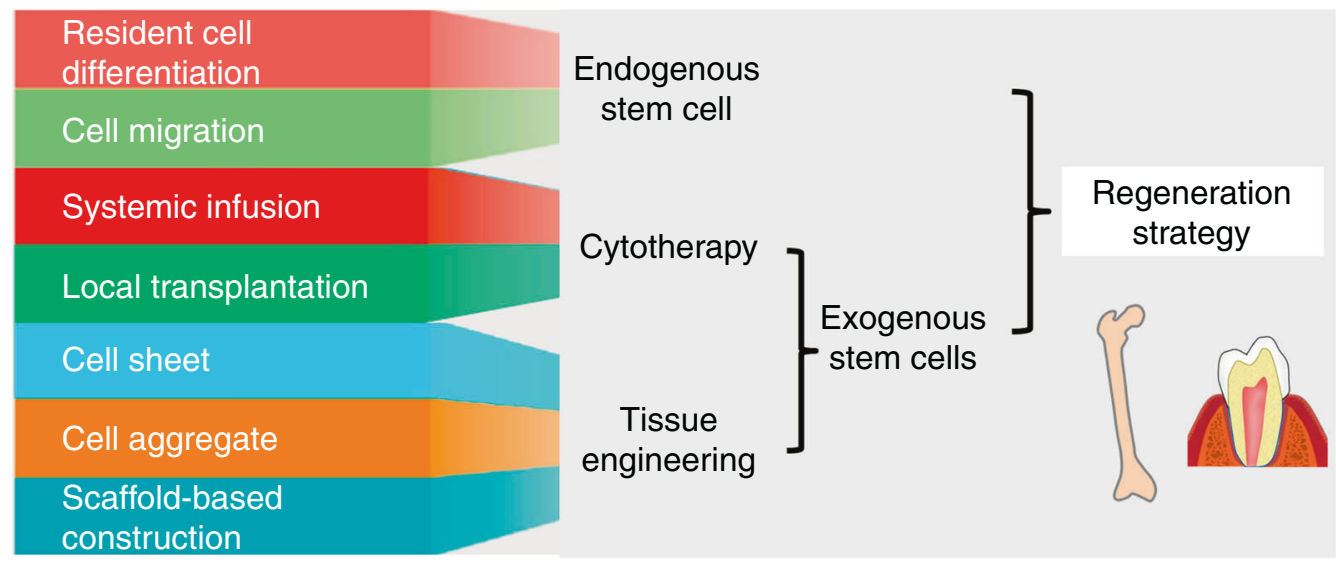

Fig. 2 Stem cell-based regenerative strategies for bone and dental tissue. Based on the cellular sources, stem cell-based bone and dental regeneration mainly includes two categories: endogenous stem cell- and exogenous stem cell-mediated regeneration strategies. Both resident and migratory endogenous stem cells have shown great potential in healing of bone and dental loss and defects. Alternatively, the application of exogenous stem cells is promising for regenerating bone and dental tissue through either cytotherapy via systemic/ locoregional infusion or tissue engineering, including cell sheet, cell aggregate and scaffold-based tissue construction

owing to neural crest or glial origins during development and subsequent fostering by the neurovascular niche during growth, makes these cells an optimal population for bone and tooth regeneration. ${ }^{71-73}$

PDLSCs. PDL is a fibrous connective tissue located between the cementum of the root and the alveolar bone socket wall, which plays an important role in supporting the teeth via anchoring the tooth to alveolar bone. ${ }^{54}$ PDLSCs, a stem cell subpopulation first discovered by Shi et al. ${ }^{74}$ from PDL, are responsible for the physical maintenance and regeneration of periodontal tissue structure and function. Upon in vitro culture, these cells possess a clonogenic nature, express a variety of cementoblastic/osteoblastic markers and are able to form mineralized nodules. Moreover, after in vivo transplantation, PDLSCs are able to form cementumand PDL-like structures. ${ }^{74}$ Regarding periodontal defects, locally transplanted PDLSCs migrated into the PDL section and successfully repaired defects, implying the potential of PDLSCs in periodontal tissue regeneration. ${ }^{75,76}$ In addition, an optimal protocol has been established with regard to the extraction, expansion and characterization of human PDLSCs, which are usually obtained from extracted orthodontic teeth or normal impacted third molars. ${ }^{18}$ Furthermore, PDLSCs can also be obtained from residual PDL on retained deciduous teeth ${ }^{77}$ or cryopreserved human $\mathrm{PDL}^{78}$ which expands the sources of PDLSCs.

SHED. In 2003, Miura et al. ${ }^{79}$ isolated a population of MSCs from the pulp tissue of the crown of exfoliated deciduous teeth differing from DPSCs and named them SHED. After in vivo implantation, SHEDs are capable of forming dentin-like structures, indicating their potential in pulp regeneration. ${ }^{79-81}$ In addition, compared with DPSCs, SHEDs exhibit higher proliferative activity, odontogenic and osteogenic differentiation potential, and osteoinductive ability. ${ }^{82,83}$ In addition, SHEDs are able to differentiate into neuronal and glial cells when cultured within neurogenic inductive media. ${ }^{79,84}$ Furthermore, SHEDs can be harvested via a relatively easy approach and maintain their regenerative potential after cryopreservation for cell banking, as demonstrated by the maintenance of surface antigens and differentiation properties after 2 years of cryopreservation. ${ }^{85}$ Remarkably, our group demonstrated that the implantation of SHED regenerated threedimensional whole dental pulp accompanied by blood vessels and nerves in both animal models and patients with tooth trauma, ${ }^{71}$ further supporting SHED as an attractive cell source for bone and tooth regeneration.
SCAP. Since apical papilla tissue only exists during root development before the tooth erupts into the oral cavity, SCAP is a unique population of DSCs located at the tips of growing tooth roots. ${ }^{86-88}$ These cells possess MSC properties, including clonogenicity and the ability to differentiate into odontoblasts/ osteoblasts in vitro. ${ }^{86,88}$ In addition, SCAP demonstrated higher proliferation rates and stronger mineralization ability in vitro than DPSCs. ${ }^{89}$ Indeed, SCAP are able to regenerate a typical cementum/ PDL-like complex in vivo, further indicating their potential for bone and dental tissue regeneration. ${ }^{90}$ In addition, SCAP have been reported to form a root/periodontal complex after cotransplantation with PDLSCs into tooth sockets of mini pigs. ${ }^{86,88}$ Moreover, cells derived from inflamed periapical tissue also exhibit typical MSC characteristics with highly osteogenic capacity in vitro and in vivo, ${ }^{91}$ suggesting the maintenance of stemness. Considering that roots develop postnatally and that the root apical papilla is clinically available from extracted wisdom teeth, SCAP can provide a source of MSCs that are isolated in the process of development and possess embryonic-like properties. ${ }^{14,54}$

DFCs. As a loose connective tissue derived from ectomesenchyme, the dental follicle surrounds the enamel and the dental papilla of the developing tooth germ before tooth eruption, which contains progenitors for osteoblasts, cementoblasts and PDL. ${ }^{92,93}$ Specifically, DFCs form the PDL via differentiation into PDL fibroblasts that secrete collagen and interrelate with fibres between the adjacent bone and cementum. DFCs are able to differentiate into cementoblasts under in vitro culture ${ }^{94,95}$ and to generate cementum when implanted in vivo. ${ }^{93}$ In addition, DFCs generated PDL-like tissue after in vivo transplantation ${ }^{96}$ and produced periodontal tissues via epithelial-mesenchymal interaction. ${ }^{97}$ Notably, DFCs maintained MSC features in culture after more than 30 passages and formed single or complex tissues in the periodontium. ${ }^{98}$ Collectively, DFCs are a promising MSC source for regenerative medicine. ${ }^{14,54}$ Furthermore, similar to SCAP, DFCs are a group of cells derived from developing tissue, thus possessing higher plasticity than other DSCs.

\section{STEM CELL-BASED REGENERATIVE STRATEGIES FOR BONE AND DENTAL TISSUE}

Regeneration with endogenous stem cells

Despite the enormous efforts devoted to exogenous MSC transplantation for tissue regeneration, an alternative therapeutic strategy is to take advantage of endogenous MSCs, which reside 


\section{Diseased microenvironment}
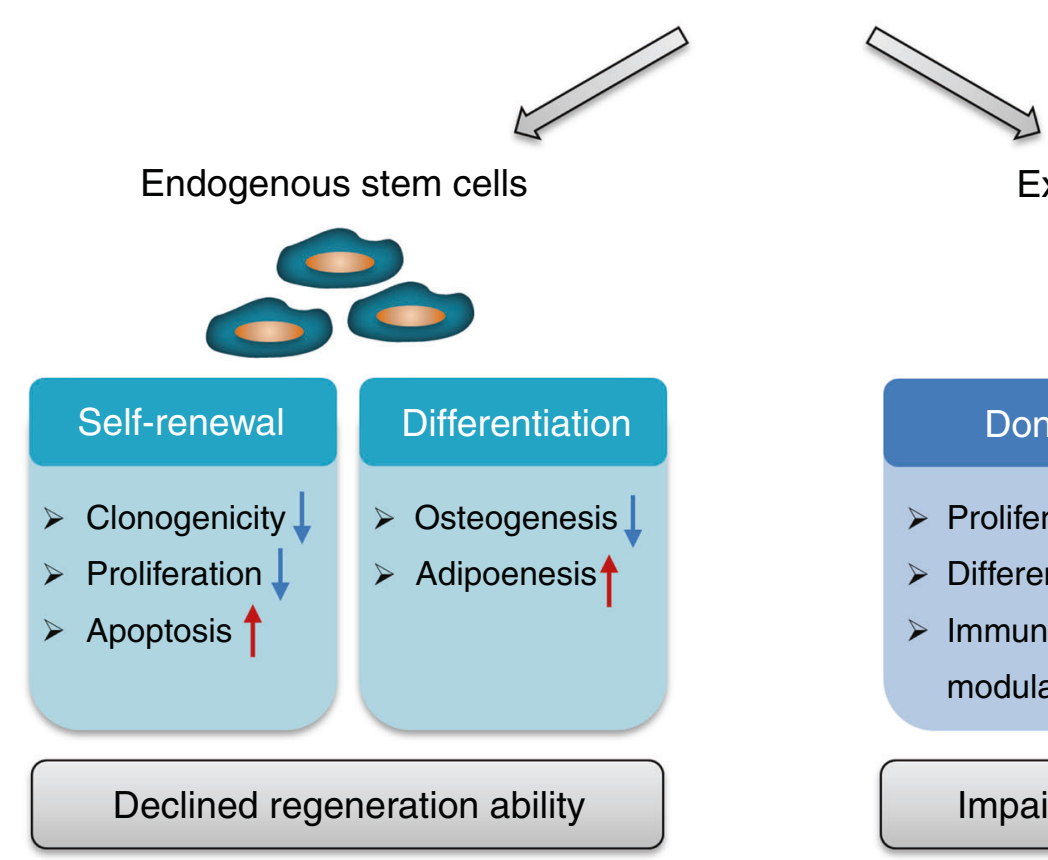

Exogenous stem cells
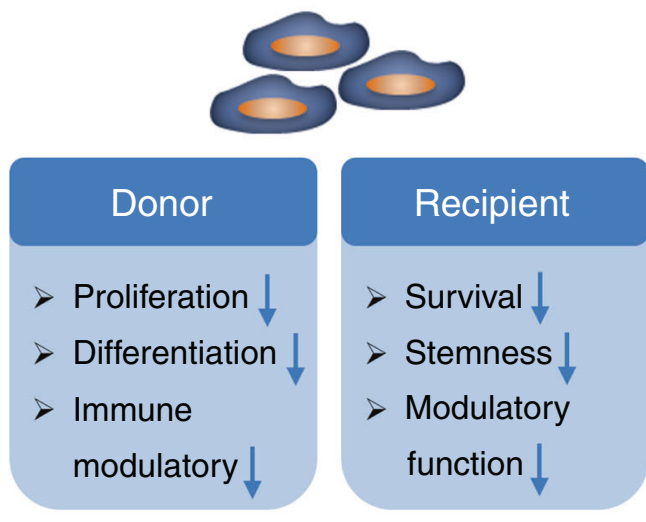

Impaired therapeutic efficacy

Fig. 3 Microenvironmental impact on stem cells in bone and dental regeneration. Under pathologic microenvironmental conditions, the survival and functions of endogenous stem cells are impaired, as shown by declined self-renewal ability and disturbed differentiation potential, leading to development of bone diseases and declined regeneration ability. On the other hand, the therapeutic efficacy of transplanted exogenous MSCs are compromised by the diseased microenvironments of the donors where they are harvested and of the recipients where they are applied due to the impairment of stemness and immune modulatory function

within specific tissues and are able to self-renew and produce specific cell types ${ }^{99-101}$ (Fig. 2). Compared with exogenous MSC transplantation, tissue regeneration mediated by endogenous MSCs is less expensive and labour intensive and avoids surgical injury and rejection risk. ${ }^{100,101}$ Nevertheless, in mammals, the regenerative capabilities of endogenous MSCs progressively decline during postnatal development, leading to limited innate repairing capacity. ${ }^{101}$ Moreover, under pathological conditions, such as osteoporosis and periodontitis, the function of endogenous MSCs is severely impaired, as characterized by decreased proliferation and osteogenic differentiation capabilities. ${ }^{21,30,31,102}$ Accordingly, pharmacological approaches have been developed to rescue MSC deficiency in bone and oral tissue loss.

Mechanistically, studies have revealed a variety of molecular mechanisms underlying gene expression regulation in bone that forms a complex signalling network, including mammalian target of rapamycin signalling, ${ }^{103,104}$ Notch signalling, ${ }^{41}$ nuclear transcription factor-kappaB (NF-KB) signalling ${ }^{105}$ and Wnt signalling, ${ }^{106}$ which suggested multiple intervention targets in restoring MSC functions. Accordingly, a variety of agents targeting the above signalling pathways have been demonstrated to be effective in rescuing endogenous MSC impairment and promoting bone and dental regeneration, such as rapamycin, ${ }^{103,104}$ DAPT, $^{41}$ PDTC, ${ }^{105}$ and dickkopf-1. ${ }^{106}$ In addition, antioxidants, including NAC, ${ }^{107-109}$ resveratrol, ${ }^{110,111}$ and melatonin, ${ }^{112}$ which protect MSCs from oxidative damage, have been successfully used to restore MSC function and improve osteopenia and periodontitis. Furthermore, approaches targeting epigenetic regulation mechanisms, such as histone modification regulators DZNep, ${ }^{113}$ pargyline ${ }^{114}$ and KDM5A, ${ }^{115}$ and microRNA expression regulation, $116-119$ have exerted therapeutic efficacy on osteoporosis, fracture healing and oral tissue regeneration. In addition to modulating in situ
MSCs, the mobilization of endogenous MSCs from other sites of the body to an injury site to replenish deficient resident MSCs also contributes to tissue regeneration. ${ }^{120-122}$ In this regard, LLP2A-Ale, which acts as a migration stimulator, has been used to accelerate bone formation in oestrogen deficiency-induced osteoporosis via directing MSCs to bone formation surfaces. ${ }^{123}$ Moreover, the combination of stromal cell-derived factor- 1 with a proteinreleasing scaffold promoted chemotaxis-induced MSC homing, leading to the repair of bone ${ }^{124}$ and periodontal ${ }^{125}$ defects. Scaffold-releasing agents to enhance endogenous MSC functions also include transforming growth factor (TGF) $-\beta 3^{126}$ and fibroblast growth factor- $2{ }^{127}$ which benefit bone and tooth regeneration. More importantly, the modulation of the specific microenvironment where stem cells reside in vivo is an effective way to regulate endogenous MSC behaviour, which will be discussed in detail in the following sections.

Regeneration with exogenous stem cells Within recent decades, the transplantation of exogenous MSCs through different routes has been widely explored in bone and dental regenerative medicine (Fig. 2). A promising strategy is the systemic application via primarily intravenous infusion and intraperitoneal delivery, which exerts therapeutic effects on various disorders, including osteoporosis, bone fracture, osteoarthritis and jaw osteonecrosis. Mechanistically, through homing to recipient bone marrow or fracture sites, infused MSCs promote osteogenesis by differentiating into osteoblasts, inducing endogenous osteoblastogenesis and modulating osteoclast-mediated bone resorption. ${ }^{49,128-130}$ In this regard, genetic or pharmacological approaches that enhance the homing of MSCs could strengthen their therapeutic efficacy on bone loss and defects. ${ }^{131-133}$ More importantly, systemically transplanted MSCs 
exhibit enormous potential to modulate systemic immunity, especially by suppressing inflammation to restore the recipient microenvironment, which also contributes to their therapeutic effects. $^{36,37,134-137}$

As alternatives to single cell suspension injection, cell sheets and aggregates provide novel strategies for cell delivery without scaffolds, enabling stable engraftment and long-term viability of transplanted cells. ${ }^{138-142}$ With the application of temperatureresponsive culture dishes, cultured cells could be non-invasively harvested as whole sheets together with their deposited extracellular matrix (ECM), which can be directly transplanted to host tissues in vivo or used to produce tissue constructs in vitro. ${ }^{138,140}$ In addition, transplanted cell sheets spatiotemporally release growth factors and modulatory cytokines, further contributing to tissue regeneration. ${ }^{54,142}$ To date, BMMSC-based cell sheets have been successfully used to promote bone regeneration in defects ${ }^{52,143}$ and strengthen implant bone bonding. ${ }^{144}$ In addition, PDLSC sheets have been demonstrated to promote periodontal regeneration in various animal species. ${ }^{145-149}$ After in vivo grafting of monolayered or layered PDLSC sheets, the cement/PDL complex was observed along with well-oriented collagen fibres, leading to the repair of periodontal defect. More importantly, clinical trials conducted on periodontitis patients further indicate the periodontal regeneration potential of PDLSC sheets. ${ }^{150,151}$

Moreover, cell aggregates produced via micro-mass pellet culture of groups of cells under certain conditions have emerged as an attractive strategy in regeneration medicine due to structural and functional similarity to native tissue. ${ }^{54,141,142}$ Compared with single cells or cell sheets, cell aggregates contain more ECM and thus may possess more biological and inductive activities. ${ }^{139,142}$ BMMSC-based aggregates could promote bone formation in a metaphyseal defect model of ovariectomized (OVX) rats, ${ }^{50}$ and PDLSC aggregates showed promise in healing periodontal defects. ${ }^{152,153}$ In addition, the application of composite cell aggregates with PDLSCs and jaw-derived BMMSCs resulted in the generation of functional PDL-like tissue both ectopically in nude mice and orthotopically in minipig. ${ }^{154}$ Notably, a clinical trial conducted by our group revealed that SHED aggregates could regenerate complete pulp tissues in patients with pulp necrosis, which were equipped with blood vessels and nerves. ${ }^{71}$ More importantly, after SHED implantation, the recipient immature permanent teeth showed increased root length and decreased apical foramina width, suggesting that the regenerated pulp acted as normal pulp with the ability to maintain continued root development. $^{71}$

The combination of cells with scaffolds and bioactive factors, which is a classical tissue engineering strategy, is also promising for bone and dental regeneration. ${ }^{3,4,6,7,14}$ Typically, bone scaffolds are made of biomaterials, including bioactive ceramics, biodegradable metals, biodegradable polymers and calcined bone, which serve as 3D structures that lead to cell migration, proliferation and differentiation. $^{22}$ Moreover, pharmacological modification of scaffolds enables the release of biological molecules, such as angiogenic factors and osteogenic factors, thus modulating the activity and function of seeded cells and endogenous cells. ${ }^{7,21,22}$ The transplantation of BMMSC sheets combined with polycaprolactone-calcium phosphate (PCL-CaP) scaffolds in vivo resulted in the formation of neo cortical and wellvascularised cancellous bone in rats. ${ }^{155}$ In addition, the application of calcined bovine bone coated with BMMSC sheets repaired critical size bone defects in osteoporotic rats. ${ }^{52}$ Moreover, PDLSC sheets combined with $\beta$-tricalcium phosphate $(\beta-T C P) /$ collagen scaffolds or $\mathrm{PCL}-\mathrm{CaP}$ scaffolds promoted periodontal regeneration with newly formed cementum and well-oriented PDL fibres. ${ }^{146,156}$ Based on current information available, an exogenous MSC-based regenerative strategy constitutes a promising approach for healing bone and dental loss and defects.

\section{MICROENVIRONMENTAL IMPACT ON STEM CELLS IN BONE} AND DENTAL REGENERATION

The microenvironment, which acts as "soil", has been increasingly recognized to exert tremendous effects on MSCs, the "seeds" in organs, especially under pathological conditions and in cell therapies (Fig. 3). As discussed above, endogenous MSCs reside in a dynamic and complex niche consisting of neighbouring cells, ECM and plentiful neurovascular bundles, which tightly control MSC behaviours. ${ }^{27,70}$ In addition, resident MSCs are modulated by the circulatory microenvironment through hormones, metabolites, inflammatory cytokines and other soluble factors. ${ }^{31}$ Notably, microenvironmental alterations play a pivotal role in the initiation and deterioration of skeletal and dental diseases. ${ }^{157}$ Under pathologic microenvironmental conditions, the survival and functions of endogenous MSCs are impaired, leading to declined regeneration capacity and extensive bone loss. ${ }^{31}$ On the other hand, the regenerative ability of transplanted exogenous MSCs is strongly influenced by the donor microenvironmental condition where they are harvested and the recipient microenvironmental niche where they home. ${ }^{21}$ Consequently, achieving therapeutic efficacy in diseased microenvironments with host comorbidities constitutes a major challenge to MSC-based regeneration at the present time. Further understanding of the impacts exerted by the diseased microenvironment on MSCs and the underlying mechanism would promote the optimization of strategies that regenerate bone and oral tissue via targeting endogenous MSCs and transplanting exogenous MSCs.

Impairment of endogenous stem cells by pathogenic microenvironment

As important systemic factors, steroid hormones, such as sex hormones (oestradiol and testosterone) and glucocorticoids, are responsible for modulating bone development and postnatal remodelling. Pathologically, hormonal disorders, particularly the dramatic decline in sex hormones in aged people and postmenopausal women, cause an imbalance between osteoblastogenesis and osteoclastogenesis, leading to the loss of bone mass and strength. Studies have demonstrated that a deficiency in oestrogen and androgen impairs the proliferation and osteogenic differentiation of BMMSCs, as shown by diminished clonogenic assay, mineral nodule formation and osteogenic marker expression. ${ }^{158-160}$ In addition, the ability of MSCs to generate new bone ectopically is also compromised by ovariectomy. ${ }^{37}$ In addition, the differentiation potential of BMMSCs is shifted towards adipocytes, leading to bone marrow fat accumulation and bone loss. ${ }^{30,161}$ Mechanistically, in addition to indirect effects via immune reactions, oestrogen could directly maintain MSC functional homoeostasis through binding to its receptors. ${ }^{162,163}$ Moreover, oestrogen deficiency causes a dramatic accumulation of reactive oxygen species (ROS), which act as an important mediator in BMMSC specification impairment ${ }^{107,160,162,163}$ and induce the apoptosis of BMMSCs. ${ }^{164}$ Recently, epigenetic mechanisms, including histone methylation, ${ }^{113}$ histone acetylation ${ }^{165}$ and microRNAs, ${ }^{119,160,161,166}$ have been revealed to also participate in oestrogen deficiency-mediated BMMSC impairment. In glucocorticoid-induced osteoporosis, the most prevalent form of secondary osteoporosis, excessive use of glucocorticoid also suppresses BMMSC proliferation and impairs the osteogenic potential of BMMSCs, resulting in bone loss. ${ }^{167}$

Another pronounced microenvironmental factor influencing bone homoeostasis is organismal metabolism. At the cellular level, the energy metabolic profiles, mainly referring to the states of glycolysis and OXPHOS, are highly influential on the fate of stem cells during development and regeneration. ${ }^{167-169}$ Notably, BMMSCs rely on glycolysis to maintain stemness while requiring glucose uptake and transformation into OXPHOS-privileged status when undergoing osteogenic differentiation. ${ }^{170,171}$ Nevertheless, excessive exposure to glucose causes BMMSC dysfunction with 
increased senescent phenotypes, ${ }^{172,173}$ which is associated with the adverse impacts of hyperglycaemia on BMMSCs, as shown in diabetic patients. Diabetes, a common metabolic disease characterized by high blood sugar, impairs multiple organ systems, including bone and oral tissue, leading to an increased risk of osteoporosis, fractures and periodontitis. ${ }^{174}$ The overall bone turnover rate is downregulated under hyperglycaemic conditions, especially bone formation parameters, as shown by reduced osteoblast numbers and decreased osteoid quantities. A pivotal mechanism underlying the diabetic osteogenesis decline is the accumulation of advanced glycation end products, which impair viability and osteogenic differentiation of BMMSCs by directly activating the receptor. ${ }^{30}$ In addition, oxidative stress also contributes to BMMSC dysfunction caused by hyperglycaemia. ${ }^{175}$ In addition, hyperglycaemia increases mitochondrial accumulation of P53, which recruits Bax and activates molecular events of apoptosis, leading to a decline of the number of BMMSCs. ${ }^{176}$ Furthermore, diabetes is an established risk factor for periodontal diseases with increased prevalence and severity. ${ }^{177}$ The multiple differentiation potential of PDLSCs derived from periodontitis patients with diabetes mellitus was dramatically impaired, ${ }^{178}$ which may involve activation of the receptor for AGEs ${ }^{179}$ and oxidative stress. $^{180}$

Despite the essential role of inflammation in bone fracture healing, a pro-inflammatory microenvironment is a pivotal pathogenetic mechanism underlying various osteopenic disorders. ${ }^{181}$ Indeed, the induction of bone loss by pro-inflammatory cytokines occurs not only in inflammatory and autoimmune diseases but also in oestrogen-deficient, hyperglycaemic and aging conditions as key secondary detrimental factors. ${ }^{104,181,182}$ In particular, inflammatory cytokines, including tumour necrosis factor- $a$ (TNF- $\alpha$ ) and interferon- $\gamma$ (IFN- $\gamma)$, synergistically induce osteogenic differentiation dysfunction of BMMSCs, ${ }^{166,182}$ which involves signalling pathways and/or epigenetic modulations. In addition, the inflammatory microenvironment causes overproduction of ROS due to the compromised function of the mitochondrial electron transport chain and antioxidant system, which also contributes to the impairment of BMMSC lineage allocation. Moreover, high levels of TNF-a have been demonstrated to induce apoptosis and inhibit the proliferation of MSCs, leading to augmented bone loss and delayed fracture healing. ${ }^{32,160}$ Furthermore, the inflammatory microenvironment acts as a key contributor to the decreased osteogenic differentiation of PDLSCs in periodontitis by intervening with the expression of signalling molecules $^{106}$ and posttranscription modulation. ${ }^{183-185}$ Intriguingly, the chronic inflammation of periodontitis impairs endoplasmic reticulum function and induces endoplasmic reticulum stress, leading to defective osteogenic differentiation of PDLSCs. ${ }^{102}$ Taken together, the diseased microenvironment plays an important role in MSC dysfunction, which provides pivotal targets for treating bone and oral diseases via rescuing endogenous MSCs.

Influence of donor microenvironment on harvested stem cells Different MSCs reside in different niches, which not only contain tissue-specific structures and components but also possess distinct properties due to contact with the systemic circulation and the external environment. In particular, oral tissues that are exposed directly to the outside environment are more prone to be affected by the surroundings. Despite the common characteristics, studies have found that MSCs derived from different sources are, to some extent, functionally distinct, leading to therapeutic discrepancies in cytotherapy. For example, compared to BMMSCs, DPSCs and SHED that are derived from neural origins are capable of differentiating into functionally active neurons and glial cells with proper environmental cues and can secrete neurotrophic factors for neuroprotection and neurite outgrowth. ${ }^{68,69}$ In this regard, DPSCs and SHED may be more advantageous for neural regeneration. In addition, SHEDs showed stronger proliferative ability than DPSCs, with higher expression of genes involved in cell proliferation and ECM formation. ${ }^{82,83}$ In addition, PDLSCs possess higher differentiation potential than gingival MSCs (GMSCs), ${ }^{186}$ while SCAP demonstrate high proliferative activity than DPSCs and PDLSCs. ${ }^{89}$ Molecularly, the discrepancy of different tissue-derived MSCs involves signalling pathways and epigenetic mechanisms. During osteogenic differentiation, Wnt signalling is more essential for BMMSCs, while the BMP pathway plays a more important role in ADMSCs, which leads to different responses of BMMSCs and ADSMCs to microRNA regulation. ${ }^{119}$ Further deciphering the distinct transcriptional and posttranscriptional regulation of different MSCs would help clarify the specificity of tissue-specific MSCs, thus promoting the development of MSC-based cell therapy.

As discussed above, a diseased microenvironment induces endogenous MSC dysfunction, which is also reflected in their therapeutic efficacy when used as exogenously transplanted MSCs. Accordingly, the influence of the donor microenvironment on MSCs is a critical issue to be considered when applying cytotherapy, especially autologous cell transplantation. Intriguingly, recent studies have revealed that under pathological conditions, different tissue-derived MSCs showed different phenotypes, which may result in discrepancies in their therapeutic efficacy. Although PDLSCs are the first choice of MSCs for periodontal regeneration, these cells are more easily impaired by the inflammatory microenvironment than GMSCs in terms of both in vitro osteogenic differentiation ability and in vivo bone formation. ${ }^{186}$ Notably, ADMSCs are more functionally stable than BMMSCs, which are prone to bone pathogenesis and display impaired regenerative potential. Unlike BMMSCs, ADMSCs derived from aged and oestrogen-deficient osteoporotic donors preserve cell viability and osteogenic differentiation potential in vitro and, more importantly, maintain regenerative abilities for bone loss and defects when transplanted in vivo. ${ }^{55-60,187,188}$ Furthermore, ADMSCs derived from OVX mice have been demonstrated to preserve anti-inflammatory capacity and alleviate bone loss in OVX recipients via systemic delivery, which implies that ADMSCs are a promising source for osteoporotic cytotherapy with resistance to a diseased microenvironment. ${ }^{59}$ Mechanistically, ADMSCs derived from osteoporotic donors maintain stemness, energy metabolism status and antioxidative defence system as well as preserved expression levels of immunomodulatory genes. ${ }^{59}$ Considering the difference between distinct MSC types in cellular properties and resistance to diseased microenvironments, evaluation and selection of MSC sources is therefore beneficial for fulfilling the therapeutic efficacy of MSC-based bone and dental regeneration.

Impact of recipient microenvironment on transplanted stem cells In recent years, studies have gradually recognized the profound effects of recipient microenvironmental status on the therapeutic efficacy of MSCs. ${ }^{21,59}$ Since it is easier for MSCs to generate new bone in immunocompromised mice, studies have revealed that the recipient pro-inflammatory $\mathrm{T}$ cells suppress the regenerative potential of MSCs through the synergistic effects of IFN- $\gamma$ and TNFa, which inhibit MSC osteogenesis and induce MSC apoptosis. ${ }^{34}$ Correspondingly, the systemic injection of T-regulatory cells (Tregs), which inhibits recipient immune responses and inflammation in immunocompetent mice, could significantly promote the repair of calvarial defects by locoregionally transplanted MSCs. ${ }^{34}$ Moreover, the modulation of transplanted MSC performance by the recipient inflammatory microenvironment also occurs in the cytotherapy of systemically infused MSCs, although with more complicating effects. The immunosuppressive function of MSCs in vivo requires the presence of recipient IFN- $\gamma$ together with other pro-inflammatory cytokines. ${ }^{35}$ Recently, researchers have revealed that recipient cytotoxic cells are essential for the initiation of MSC-induced immunosuppression. ${ }^{189}$ Nevertheless, 

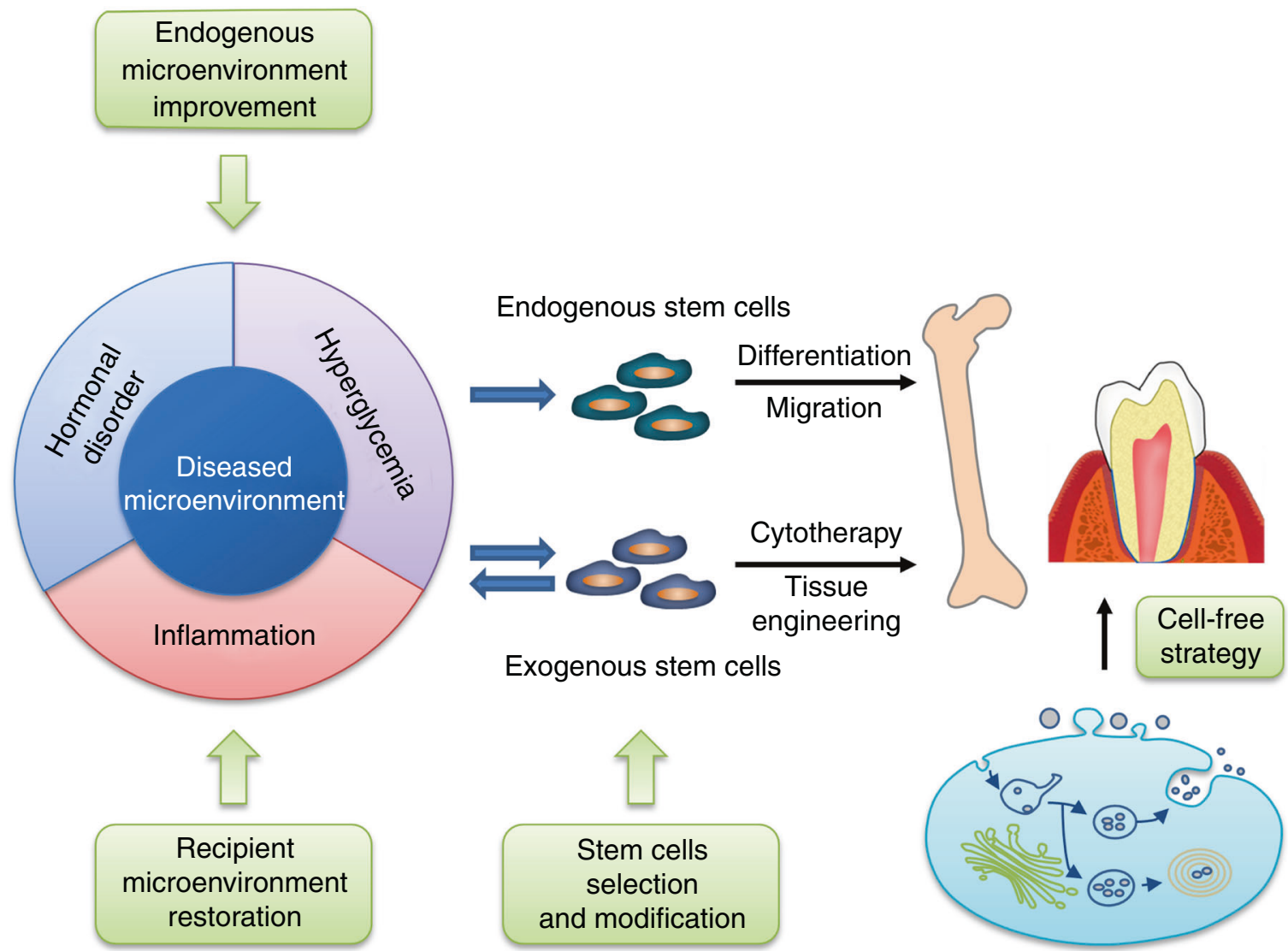

Fig. 4 Stem cell-based bone and dental regeneration: a view of microenvironmental modulation. Diseased microenvironments, such as hormonal disorders, hyperglycaemia and inflammation, severely impair the therapeutic efficacy of both endogenous and exogenous stem cell-based regeneration of bone and dental tissue. Accordingly, microenvironmental therapeutics, including improvement of the endogenous niche, selection and modification of donor stem cells, and restoration of recipient microenvironment, have shown great promises in optimizing stem cell-based bone and dental regeneration. Furthermore, extracellular vesicles are emerging as promising cell-free strategies for bone and dental regeneration

under excessive inflammation conditions, such as the arthritic milieu, the immunomodulatory ability of MSCs is abrogated, leading to therapeutic failure. ${ }^{190}$ Accordingly, it is likely that a balanced recipient inflammatory microenvironment is pivotal to elicit MSC-mediated immunomodulation and bone healing, which is the case for osteoporotic models such as ovariectomy and systemic lupus erythaematosus (SLE). ${ }^{37,136}$

Considering that osteoporotic remedy is not always achieved even under an inflammatory microenvironment, it is possible that other microenvironmental factors also control the therapeutic effects of MSCs on bone and dental disorders. Given that diabetes is a high risk factor for bone and dental diseases and that hyperglycaemia severely impairs the function of endogenous MSCs, our group further elucidated the influence of the recipient glycaemic microenvironment on cytotherapy mediated by transplanted MSCs. We found that recipient diabetes hinders the therapeutic efficacy of systemically injected MSCs on osteoporosis, which indicates that the metabolic status of the recipient microenvironments also constitutes a critical factor determining the efficacy of MSC-based therapies. ${ }^{33}$ Accordingly, the injection of insulin to restore recipient glucose homoeostasis before the infusion of MSCs could recover the therapeutic effects of MSCs to treat diabetic osteopenia. ${ }^{33}$ Molecularly, adenosine monophosphate-activated protein kinase signalling is involved in the impairment of MSC-mediated immunomodulation by recipient hyperglycaemia. ${ }^{33}$ Taken together, the recipient microenvironment plays a pivotal role in controlling MSC-mediated regenerative therapy, which needs to be further elucidated to promote the development of broadly applicable strategies for bone and dental tissue regeneration.

\section{STRATEGIES TO OPTIMIZE STEM CELL-BASED BONE AND DENTAL REGENERATION VIA TARGETING THE MICROENVIRONMENT}

Despite the enormous advancement achieved in recent years, stem cell-based bone and tooth regeneration are still faced with many challenges, especially low and uncertain efficacy. $8,14,15,22$ Microenvironments play a critical role in controlling both endogenous and exogenous MSC-mediated healing of bone and dental loss and defects, which underlies the failure of MSCbased therapies in diseased microenvironments. ${ }^{21}$ Accordingly, we envisage that strategies targeting the microenvironment will greatly promote the fulfilment of MSC therapeutic potential (Fig. 4). On the one hand, approaches aimed at correcting the diseased microenvironment could facilitate endogenous MSC-based regeneration. ${ }^{24,101}$ On the other hand, enhancing the resistance of MSCs to diseased microenvironment and/or pre-normalizing recipient microenvironment would enhance the therapeutic efficacy of transplanted exogenous MSCs. ${ }^{21}$ In addition, considering that scaffolds act as niches for seeded cells, modification of biomaterial-based scaffolds to recreate the specific and instructive microenvironment is beneficial for MSC-based tissue engineering. ${ }^{6}$ 
Improvement of the microenvironment to restore endogenous stem cell function

Considering the aetiological role of diseased microenvironmentinduced MSC dysfunction in bone and dental disorders, a promising strategy to facilitate endogenous MSC-based bone and tooth repair is to manipulate the stem cell microenvironment, which is less expensive and labour intensive and avoids surgical injury and rejection risk. Recently, tantalizing evidence has emerged that therapeutics via microenvironmental interventions could potently restore normal host conditions that rejuvenate endogenous MSCs for bone and dental healing. The application of hormonal replacement therapy for postmenopausal women with oestrogen or a combination of oestrogen and progestogen is protective against osteoporosis, ${ }^{191}$ which is in part due to the restoration of MSC functions. Specifically, the osteogenic potential of MSCs derived from osteoporotic patients can be promoted by oestrogen treatment but not by testosterone, as shown by increased calcium deposition and osteogenic gene expression, ${ }^{162}$ indicating a critical role for oestrogen in maintaining MSC functions. In addition to improving MSC-mediated osteogenesis, supplementation with oestrogen also enhances MSC-induced apoptosis of osteoclasts by preserving Fas ligand (Fasl) expression. ${ }^{192}$ Further studies are needed to establish medication usage to mimic the physical pattern of hormone secretion. ${ }^{191}$

Recently, caloric restriction that lowers glucose metabolism and nutrient intake has been increasingly recognized as a rejuvenative intervention in many organisms to rescue functional decline in various human organs. ${ }^{193}$ Notably, life-long caloric restriction has been demonstrated to prevent age-induced bone loss, while short-term caloric restriction exerted no effects. ${ }^{194,195}$ For MSCs, the downregulation of glucose levels in the in vitro culture microenvironment dramatically protected MSCs from replicative senescence after serial passages with strengthened proliferation and osteogenic potential. ${ }^{172}$ In addition, metabolic control of hyperglycaemia via the long-term infusion of insulin preserved bone mineral density and lowered fracture risk in diabetic patients. ${ }^{196,197}$ Moreover, pharmacological intervention by metabolic regulators has been reported to restore impairment of BMMSC-mediated osteoblastogenesis and alleviate bone disorders. For example, treatment with resveratrol via oral feeding or intraperitoneal injection alleviated the in vivo skeletal senescent phenotype with improvement of resident BMMSCs osteogenic potential. ${ }^{110,198}$ In addition, the administration of resveratrol restored ligature/lipopolysaccharide-induced alveolar bone loss, with inhibition of inflammation and oxidative stress. ${ }^{111}$ Moreover, the administration of metformin in drinking water or through local injection promoted BMMSC osteogenic differentiation, resulting in facilitation of bone lesion regeneration in diabetic rats ${ }^{199}$ and prevention of bone ageing. ${ }^{200}$

The above findings have highlighted the prevalence of inflammation in bone and dental disorders and the impairment of MSCs by the pro-inflammatory microenvironment. Accordingly, anti-inflammation therapy constitutes an important approach for bone and dental regeneration via restoring endogenous MSC functions. Indeed, genetic deletion of TNF-a exerts protective effects on bone mass under diseased conditions. ${ }^{201}$ At the cellular level, the impaired osteogenic differentiation ability of BMMSCs derived from oestrogen-deficient mice was rescued by treatment with neutralizing antibodies that deplete either TNF-a or IFN- $-{ }^{182}$ Further in vivo infusion of these antibodies also rescued resident BMMSC dysfunction and alleviated OVX-induced osteoporosis, with enhanced effects when used together. ${ }^{182}$ Notably, the application of anti-TNF-a at earlier and later stages of oestrogen deficiency-induced osteoporosis exerted preventive and curative effects on bone loss, respectively. ${ }^{166}$ Pharmacologically, antiinflammatory drugs have been demonstrated to improve bone and dental diseases via rescuing resident MSC homoeostasis. ${ }^{183,202}$ Systemic administration of aspirin via oral feeding downregulated the systemic concentration of TNF- $a$ and IFN- $\gamma$, thus restoring MSC functions and abrogating oestrogen-deficientinduced bone loss. ${ }^{182,202}$ Furthermore, aspirin treatment restored deficient osteogenic differentiation of PDSLCs under an inflammatory microenvironment, which upon in vivo injection, rescued periodontitis. ${ }^{183}$ Clinical translation of these microenvironmental therapies will help optimize endogenous MSC-based bone and dental regeneration.

Cell source evaluation and cellular modification to obtain feasible stem cells

As discussed above, different sources of MSCs display distinct resistance to diseased microenvironments, suggesting that the selection of cell sources is essential for optimizing regenerative therapies. To date, BMMSCs are the most popular candidates in bone regeneration therapy both via cytotherapy and tissue engineering. ${ }^{3}$ However, BMMSC functions are prone to a diseased skeletal microenvironment, leading to therapeutic efficacy impairment of autologous cells. ${ }^{60,77}$ In contrast, ADMSCs derived from bone pathological donors (i.e. ageing or oestrogen deficiency) preserve functional homoeostasis and therapeutic effects in locoregional bone regeneration and systemic cytotherapy for osteoporosis, indicating more resistance to a diseased microenvironment than BMMSCs. ${ }^{55-61,187,188}$ In addition, PDLSCs, which are recognized as the standard stem cell source for periodontal regeneration, are also more vulnerable to pro-inflammatory microenvironments than GMSCs. ${ }^{186}$ Therefore, further elucidation of the discrepancy among different MSC types could provide better knowledge on how to select cell sources, especially for autologous cellular therapy.

In addition to innate divergence between different MSC populations, pre-conditioning provides an efficacious approach to enhance MSC resistance to the diseased recipient microenvironment and improve MSC-based bone and dental regeneration. Genetic modification may enormously affect MSC functions via changing the expression of key genes involved in stem cell properties, which, however, may not be suitable for clinical application. ${ }^{131,133}$ Alternatively, epigenetic reprogramming via treatment with posttranscriptional modifiers has shown promise in achieving long-lasting resistance of MSCs to recipient microenvironmental impacts. ${ }^{113,119}$ For example, miR-26a overexpression rescued the impaired capacity of oestrogen-deficient mousederived MSCs in ectopic bone formation and in healing criticalsized calvarial bone defects. ${ }^{119}$ In addition, the histone methylation inhibitor DZNep enhanced MSC osteogenic potential under in vitro pathogenic conditions, ${ }^{113}$ which need further studies to evaluate the in vivo effects. On the other hand, pharmacological intervention with small molecule compounds has shown promise in enhancing MSC regenerative potential. Pretreating MSCs with aspirin before in vivo transplantation enhanced MSC resistance to the recipient inflammatory microenvironment, resulting in significant improvement of MSC-based ectopic bone regeneration. $^{34,203}$ In addition, melatonin treatment promoted MSC osteogenesis abilities, resulting in enhanced local bone regeneration mediated by MSCs both in ectopic sites and in critical-sized calvarial bone defects. ${ }^{204}$ Notably, pre-conditioning with melatonin strengthened the therapeutic effects of systemically transplanted MSCs for OVX-induced osteoporosis with restoration of recipient bone remodelling, indicating an enhancement of their resistance to oestrogen-deficient and inflammatory microenvironments. $^{204}$ In addition, it has been demonstrated that metformin pretreatment helped maintain MSC immunomodulation ability under high glucose conditions, leading to preservation of MSC therapeutic efficacy on osteoporosis with hyperglycaemia. ${ }^{33}$ Intriguingly, a low concentration of IFN- $\gamma$ has been demonstrated to be essential for priming donor MSC immunomodulation property $^{35,205}$ and to promote MSC-based bone formation. ${ }^{206}$ With the above approaches, the ability of MSCs to resist diseased 
Table 1. Clinical trials of stem cell-based therapies for dental regeneration

\begin{tabular}{|c|c|c|c|c|}
\hline Indication & Cell source & Phase, patients & Results/status & Clinical trial \\
\hline & DPSCs & $\mathrm{N} / \mathrm{A}, n=30$ & Completed (no results posted) & NCT02842515 \\
\hline Periapical periodontitis & Umbilical cord-derived MSCs & $\mathrm{N} / \mathrm{A}, n=38$ & Completed (no results posted) & NCT03102879 \\
\hline \multirow[t]{6}{*}{ Periodontitis } & DPSCs & $\mathrm{N} / \mathrm{A}, n=29$ & Completed (no results posted) & NCT03386877 \\
\hline & & Phase $1 / 2, n=40$ & Unknown status & NCT02523651 \\
\hline & & Phase $1 / 2, n=80$ & Unknown status & NCT01082822 \\
\hline & BMMSCs & Phase $1 / 2, n=30$ & Completed (no results posted) & NCT02449005 \\
\hline & GMSCs & Phase $1 / 2, n=30$ & Recruiting & NCT03137979 \\
\hline & MSCs & Phase $1 / 2, n=10$ & Completed (no results posted) & NCT00221130 \\
\hline Alveolar bone loss & DPSCs & Phase $1, n=10$ & Enrolling by invitation & NCT02731586 \\
\hline \multirow[t]{2}{*}{ Cleft lip and palate } & DPSCs & Phase $3, n=62$ & Not yet recruiting & NCT03766217 \\
\hline & & $\mathrm{NA}, n=5$ & Completed (satisfactory bone healing) & NCT01932164 \\
\hline Cleft of alveolar ridge & Buccal fat pad derived stem cells & Phase $1, n=10$ & Completed (no results posted) & NCT02859025 \\
\hline Jaw bone atrophy & MSCs & Phase $1, n=13$ & Enrolling by invitation & NCT02751125 \\
\hline
\end{tabular}

microenvironments will be strengthened, which helps guarantee the therapeutic efficacy of these cells.

Restoration of recipient microenvironment to benefit transplanted stem cells

Considering the role of recipient microenvironments in determining the therapeutic efficacy of transplanted stem cells, prenormalizing the microenvironment to provide a favourable regeneration condition is another promising strategy to optimize MSC-based bone and tooth regeneration. Before local transplantation of MSCs into the injury area, the application of the antiinflammatory agent aspirin specifically around the transplantation location inhibited the regional pro-inflammatory condition with downregulation of IFN- $\gamma$ and TNF- $a$. As a result, MSC-mediated repair of critical-sized calvarial bone defects was promoted. ${ }^{34}$ In addition, for MSC-based systemic cytotherapy, the short-term application of a proteasome inhibitor, bortezomib, at arthritis onset enhanced MSC therapeutic efficacy for arthritis via inhibiting the diseased inflammatory microenvironment. ${ }^{190}$ Moreover, for diabetic recipients, the therapeutic effects of MSCs may be promoted by controlling the hyperglycaemic microenvironment. During systemic MSC injection, intensive infusion of insulin, which helped maintain a normal condition, guaranteed the effects of MSCs to treat osteopenia. ${ }^{33}$ Notably, the one-time injection of insulin at a low dose just prior to MSC infusion could enable MSCs to alleviate osteoporosis in diabetic recipients, which indicated the feasibility of building up a normoglycaemic "window" for MSC transplantation. ${ }^{33}$

Another approach to normalize the diseased recipient microenvironment is through the delivery of cells. For MSC-mediated ectopic bone regeneration, infusion of Tregs modulated the recipient inflammatory microenvironment, leading to improved regeneration in immunocompetent recipients. ${ }^{34}$ Notably, MSCs are able to modulate the recipient microenvironmental condition via cell-cell contact and paracrine secretion of a variety of cytokines and EVs. ${ }^{35,36,41}$ Indeed, MSC-mediated bone healing via locoregional transplantation could be enhanced by synchronized systemic MSC infusion, which inhibited recipient immunological responses and decreased inflammatory levels. ${ }^{135}$ Similarly, under certain conditions, multiple systemic infusions of MSCs have superior effects to one-time MSC infusion due to the modulatory effects of MSCs. ${ }^{207,208}$ Moreover, the two-time infusion of MSCs has been proven as effective in the treatment of diabetic osteoporosis, during which the first restored normoglycaemic conditions to create a beneficial microenvironment so that the second infused MSCs could ameliorate osteopenia. ${ }^{33}$

In addition to cytotherapy, establishing a favourable microenvironment is also an effective approach to promote the regenerative efficacy of MSC-based tissue engineering. The cell sheet/aggregate technique, which delivers cells with intact surface adhesion molecules and cell-cell interactions, has been recognized to establish a beneficial microenvironment for exogenously transplanted MSCs. ${ }^{209}$ Moreover, the bone regeneration potential of MSC aggregates could be enhanced by licochalcone $A$, a small molecular compound promoting ECM secretion and MSC osteogenic differentiation, resulting in facilitated healing of metaphyseal defects in oestrogen-deficient recipients. ${ }^{50}$ On the other hand, bioactive biomaterials with the ability to create a beneficial ambient microenvironment have been increasingly applied in tissue engineering along with bioactive molecular modification. The application of strontium-substituted calcium silicate bioactive ceramic scaffolds facilitated bone regeneration in osteoporotic recipients. ${ }^{210}$ In addition, akermanite bioceramics significantly promoted the healing of critical-sized calvarial defects in oestrogen-deficient mice and that calcium, magnesium and silicon-containing akermanite bioceramics enhanced the osteogenesis and angiogenesis of OVX mice-derived MSCs. ${ }^{211}$ Furthermore, mesoporous bioglass/silk fibrin scaffolds combined with delivery of platelet-derived growth factor $B$ and bone morphogenetic protein-7 (BMP-7) have shown notable pro-regenerative effects on osteoporotic femoral defects. ${ }^{212}$ In addition, the use of calcium phosphate cement scaffolds loaded with icariin could create a beneficial niche in oestrogen-deficient conditions to increase the osteogenesis and angiogenesis of MSCs, thus promoting MSC-based osteoporotic fracture healing. ${ }^{213}$ Taken together, the restoration of recipient microenvironments is a 
10

feasible way to optimize MSC-based bone and dental regeneration.

CELL-FREE STRATEGIES IN BONE AND DENTAL REGENERATION As discussed above, MSCs possess a strong ability to secrete EVs containing a wide variety of proteins, lipids, and nucleic acids, which play a key role in mediating cell-cell communication. Notably, EVs have emerged as a promising alternative to wholecell therapy with considerable potential in bone and dental regeneration. ${ }^{214-216}$ Exosomes, the most extensively studied EV type, have been increasingly recognized as the main components of the secretome mediating MSC therapeutic effects on osteoporosis, the systemic injection of which alleviates osteopenia as efficiently as MSCs. ${ }^{39,41}$ In particular, both MSCs and exosomes exert long-lasing therapeutic effects, with one-time injection resulting in restoration of bone mass for months. ${ }^{39,41}$ The underlying mechanism may be the modulation of recipient epigenetic states via transfer of microRNAs and proteins. ${ }^{39,41}$ In addition, the application of MSC-derived exosomes alone or combined with scaffolds has exhibited the potential to promote bone regeneration for fracture, defects, osteoarthritis and osteonecrosis. ${ }^{40,217-221}$ Furthermore, exosomes exhibit preserved therapeutic efficacy in a diseased microenvironment, as shown in the healing of critical-sized bone defects in OVX rats. ${ }^{222}$ The ability of exosomes to resist recipient pathogenic microenvironments has been further proven, as these molecules maintain therapeutic potential for osteoporosis under autoimmune conditions, such as SLE and systemic sclerosis. ${ }^{39,41}$ Given the diversity of EVs, further elucidation of the therapeutic prospects of other EVs is needed, which will promote the establishment of cell-free regeneration strategies.

Within recent years, EV-based cell-free strategies have shown encouraging therapeutic potential with superiority to single molecule drugs, whole cells, and synthetic liposome or nanoparticle formulations. EVs are easily obtained and stored sustainably and reproducibly and remain relatively stable when infused in vivo. In addition, by transferring a variety of secreted factors, EVs are able to exert synthetical effects on the recipient microenvironment with high safety. In addition, the use of engineered EVs enables the application of multiple factors with favourable biocompatibility and biostability and shows less risks for differentiation abnormalities or neoplastic transformation. Collectively, the translation of MSC-derived EVs into clinically feasible therapeutics will become a thriving strategy in bone and dental regeneration, which will stimulate an enormous amount of preclinical and clinical researches in the near future.

\section{CLINICAL TRIALS OF MSC-BASED BONE AND DENTAL REGENERATION}

In recent years, an increasing number of clinical trials have been conducted to assess the clinical feasibility of MSC-based regenerative therapies for bone and dental diseases (Table 1). Uncontrolled clinical trials have demonstrated that the intraarticular injection of ADMSCs is a safe and effective therapeutic approach for patients with knee osteoarthritis that significantly improves pain, function and daily living activities. ${ }^{223}$ For the treatment of osteonecrosis of the femoral head (ONFH), the application of bone marrow-derived mononuclear cells (BMMCs) that are highly enriched with BMMSCs leads to a notable alleviation of clinical symptoms, improved hip function and delayed collapse, thus constituting a safe, efficacious and minimally invasive treatment approach, especially for patients at early stages of ONFH. ${ }^{224-227}$ In addition, in one clinical study, patients with calvarial defects underwent a cranioplasty procedure using a combination of ADMSCs and beta-tricalcium phosphate granules, which promotes reconstruction of large cranial defects. $^{228}$ In addition, the clinical relevance of MSCs in dental regeneration has also been assessed. In a randomized controlled clinical study conducted by our group, SHED aggregates could regenerate complete pulp tissues in patients with pulp necrosis, which is equipped with blood vessels and nerves. ${ }^{71}$ More importantly, after SHED implantation, the recipient immature permanent teeth showed increased root length and decreased apical foramina width, indicating that the regenerated pulp acted as normal pulp to maintain continued root development. ${ }^{71}$ For patients with severe mandibular ridge resorption, researchers inserted BMMSCs into the resorbed alveolar ridge together with biphasic calcium phosphate granules as scaffolds, which induced significant new bone formation that was adequate for dental implant installation. ${ }^{229}$ Furthermore, a single-centre, randomized trial was conducted to assess the efficacy of PDLSCs in combination with bovine-derived bone mineral materials for the treatment of periodontal intrabony defects. No statistically significant differences were detected between the experimental group and the control group, which needs to be further validated by multicentre randomized controlled studies. ${ }^{230}$

However, despite the promising results of these studies, there are still many obstacles limiting the use of MSCs in clinical bone and dental regeneration. Many of the completed clinical trials registered in ClinicalTrials.gov have not provided results, which may restrain the clinical transformation of MSC-based regenerative therapies. In addition, the development of internationally recognized, standardized guidelines on cell selection, expansion, storage and shipping are needed to provide clinically applicable cell sources. Another aspect that needs to be addressed is the lack of a standardized procedure for cytotherapy or the application of MSC-based tissue engineering products. More importantly, the fulfilment of the function of transplanted cells requires technological advances that optimize the retention, viability, homing, differentiation ability and modulatory capacity of MSCs in vivo.

\section{CONCLUSION}

Over the past several years, MSC-based regeneration strategies have shown great promise for healing bone and dental loss and defects, both via endogenous restoration and exogenous transplantation. Notably, the therapeutic efficacy of MSC-mediated regeneration is under tight control of the microenvironment, which not only regulates resident MSCs under both physical and pathological conditions but also modulates transplanted MSCs in cytotherapy and tissue engineering. As a result, achieving MSCbased bone and dental regeneration in diseased microenvironments remains a major challenge. Accordingly, microenvironmenttargeting therapeutic strategies that may promote the optimization of MSC-based bone and dental healing in diseased microenvironments have been established. In this regard, several tactics have demonstrated enormous potential, including improvement of the endogenous microenvironment to revitalize innate MSCs, modification via pharmacological or epigenetic approaches to enhance exogenous MSC resistance, and restoration of the recipient microenvironment to benefit transplanted MSCs. Notably, EVs/exosomes have emerged as attractive alternatives to MSCs in both cytotherapy and tissue engineering with pro-regenerative potential and microenvironment modulatory abilities (Fig. 4).

While much progress has been achieved, several issues remain to be explored. First, further studies regarding the microenvironmental modulation of MSC-based tissue regeneration and underlying molecular mechanisms are needed to pinpoint the specific contributions of the microenvironment to MSC-based therapies and identify key molecules and signalling pathways involved. Second, the application of novel techniques to improve MSCbased bone and dental regeneration, such as modifying biomimicking materials via nanotechnology to establish a bionic 
microenvironment ${ }^{231-233}$ and strengthening MSC recruitment via an aptamer-targeting technique to promote oriented transplantation, is needed. ${ }^{234,235}$ Third, given the control of the microenvironment over MSCs, it is advisable to analyse the recipient microenvironment status and accordingly formulate therapeutic time points prior to MSC transplantation to strengthen the efficacy of infused MSCs. Last but not least, in addition to prolonging the survival of transplanted MSCs, recent studies have revealed that the apoptosis of MSCs may constitute a critical mechanism underlying their therapeutic efficacy in certain disorders, ${ }^{188,236}$ which may provide novel insights into MSC-based regenerative therapies. In summary, understanding the effects of microenvironmental modulation on MSCs will shed more light on the pathogenesis and therapeutics of bone and tooth, which will promote the establishment of optimized MSC-based strategies for bone and dental regeneration.

\section{ACKNOWLEDGEMENTS}

This work was supported by the National Key Research and Development Program of China (2016YFC1101400 to Y.J.) and the National Natural Science Foundation of China (31800817 to S.L., 31870970 to J.Z.).

\section{ADDITIONAL INFORMATION}

Competing interests: The authors declare no competing interests.

\section{REFERENCES}

1. Gong, T. et al. Nanomaterials and bone regeneration. Bone Res. 3, 15029 (2015).

2. Zhou, X. et al. Oral health in China: from vision to action. Int. J. Oral. Sci. 10, 1 (2018).

3. Fernandes, G. \& Yang, S. Application of platelet-rich plasma with stem cells in bone and periodontal tissue engineering. Bone Res. 4, 16036 (2016).

4. Henkel, J. et al. Bone regeneration based on tissue engineering conceptions-a 21st century perspective. Bone Res. 1, 216-248 (2013).

5. Kinane, D. F., Stathopoulou, P. G. \& Papapanou, P. N. Periodontal diseases. Nat. Rev. Dis. Primers 3, 17038 (2017).

6. Ho-Shui-Ling, A. et al. Bone regeneration strategies: engineered scaffolds, bioactive molecules and stem cells current stage and future perspectives. Biomaterials 180, 143-162 (2018).

7. Bose, S., Roy, M. \& Bandyopadhyay, A. Recent advances in bone tissue engineering scaffolds. Trends Biotechnol. 30, 546-554 (2012).

8. Moioli, E. K., Clark, P. A., Xin, X., Lal, S. \& Mao, J. J. Matrices and scaffolds for drug delivery in dental, oral and craniofacial tissue engineering. Adv. Drug Deliv. Rev. 59, 308-324 (2007).

9. Bianco, P. et al. The meaning, the sense and the significance: translating the science of mesenchymal stem cells into medicine. Nat. Med. 19, 35-42 (2013).

10. Frenette, P. S., Pinho, S., Lucas, D. \& Scheiermann, C. Mesenchymal stem cell: keystone of the hematopoietic stem cell niche and a stepping-stone for regenerative medicine. Annu. Rev. Immunol. 31, 285-316 (2013).

11. Uccelli, A., Moretta, L. \& Pistoia, V. Mesenchymal stem cells in health and disease. Nat. Rev. Immunol. 8, 726-736 (2008).

12. Daley, G. Q. The promise and perils of stem cell therapeutics. Cell Stem Cell 10, 740-749 (2012).

13. Trounson, A. \& McDonald, C. Stem cell therapies in clinical trials: progress and challenges. Cell Stem Cell 17, 11-22 (2015).

14. Volponi, A. A., Pang, Y. \& Sharpe, P. T. Stem cell-based biological tooth repair and regeneration. Trends Cell Biol. 20, 715-722 (2010).

15. Grayson, W. L. et al. Stromal cells and stem cells in clinical bone regeneration. Nat. Rev. Endocrinol. 11, 140-150 (2015).

16. Grottkau, B. E. \& Lin, Y. Osteogenesis of adipose-derived stem cells. Bone Res. 1, 133-145 (2013).

17. Bacakova, L. et al. Stem cells: their source, potency and use in regenerative therapies with focus on adipose-derived stem cells-a review. Biotechnol. Adv. 36, 1111-1126 (2018).

18. Zhai, Q., Dong, Z., Wang, W., Li, B. \& Jin, Y. Dental stem cell and dental tissue regeneration. Front. Med. 13, 152-159 (2018).

19. Hu, L., Liu, Y. \& Wang, S. Stem cell-based tooth and periodontal regeneration. Oral. Dis. 24, 696-705 (2018).
20. Wang, Y., Chen, X., Cao, W. \& Shi, Y. Plasticity of mesenchymal stem cells in immunomodulation: pathological and therapeutic implications. Nat. Immunol. 15, 1009-1016 (2014).

21. Sui, B. D. et al. Stem cell-based bone regeneration in diseased microenvironments: challenges and solutions. Biomaterials 196, 18-30 (2017).

22. Gao, C., Peng, S., Feng, P. \& Shuai, C. Bone biomaterials and interactions with stem cells. Bone Res. 5, 17059 (2017).

23. Loeffler, J., Duda, G. N., Sass, F. A. \& Dienelt, A. The metabolic microenvironment steers bone tissue regeneration. Trends Endocrinol. Metab. 29, 99-110 (2018).

24. Lane, S. W., Williams, D. A. \& Watt, F. M. Modulating the stem cell niche for tissue regeneration. Nat. Biotechnol. 32, 795-803 (2014).

25. Wagers, A. J. The stem cell niche in regenerative medicine. Cell Stem Cell 10, 362-369 (2012).

26. Watt, F. M. \& Hogan, B. L. Out of Eden: stem cells and their niches. Science 287, 1427-1430 (2000)

27. Crisan, M. et al. A perivascular origin for mesenchymal stem cells in multiple human organs. Cell Stem Cell 3, 301-313 (2008).

28. Voog, J. \& Jones, D. L. Stem cells and the niche: a dynamic duo. Cell Stem Cell 6, 103-115 (2010).

29. Zhang, J. et al. The effect of aging on the pluripotential capacity and regenerative potential of human periodontal ligament stem cells. Biomaterials $\mathbf{3 3}$, 6974-6986 (2012).

30. Sui, B. et al. Mesenchymal progenitors in osteopenias of diverse pathologies: differential characteristics in the common shift from osteoblastogenesis to adipogenesis. Sci. Rep. 6, 30186 (2016).

31. Sui, B. D., Hu, C. H., Zheng, C. X. \& Jin, Y. Microenvironmental views on mesenchymal stem cell differentiation in aging. J. Dent. Res. 95, 1333-1340 (2016).

32. Ko, K. I. et al. Diabetes reduces mesenchymal stem cells in fracture healing through a TNFalpha-mediated mechanism. Diabetologia 58, 633-642 (2015).

33. Sui, B. D. et al. Recipient glycemic micro-environments govern therapeutic effects of mesenchymal stem cell infusion on osteopenia. Theranostics 7, 1225-1244 (2017).

34. Liu, Y. et al. Mesenchymal stem cell-based tissue regeneration is governed by recipient $\mathrm{T}$ lymphocytes via IFN-gamma and TNF-alpha. Nat. Med. 17, 1594-1601 (2011)

35. Ren, G. et al. Mesenchymal stem cell-mediated immunosuppression occurs via concerted action of chemokines and nitric oxide. Cell Stem Cell 2, 141-150 (2008).

36. Akiyama, K. et al. Mesenchymal-stem-cell-induced immunoregulation involves FAS-ligand-/FAS-mediated T cell apoptosis. Cell Stem Cell 10, 544-555 (2012).

37. Liu, Y. et al. Transplantation of SHED prevents bone loss in the early phase of ovariectomy-induced osteoporosis. J. Dent. Res. 93, 1124-1132 (2014).

38. Todeschi, M. R. et al. Transplanted umbilical cord mesenchymal stem cells modify the in vivo microenvironment enhancing angiogenesis and leading to bone regeneration. Stem Cells Dev. 24, 1570-1581 (2015).

39. Chen, $C$. et al. Mesenchymal stem cell transplantation in tight-skin mice identifies miR-151-5p as a therapeutic target for systemic sclerosis. Cell Res. 27, 559-577 (2017).

40. Furuta, T. et al. Mesenchymal stem cell-derived exosomes promote fracture healing in a mouse model. Stem Cells Transl. Med. 5, 1620-1630 (2016).

41. Liu, S. et al. MSC transplantation improves osteopenia via epigenetic regulation of notch signaling in lupus. Cell Metab. 22, 606-618 (2015).

42. Berry, D. C., Jiang, Y. \& Graff, J. M. Emerging roles of adipose progenitor cells in tissue development, homeostasis, expansion and thermogenesis. Trends Endocrinol. Metab. 27, 574-585 (2016).

43. Aicher, W. K. et al. Regeneration of cartilage and bone by defined subsets of mesenchymal stromal cells-potential and pitfalls. Adv. Drug Deliv. Rev. 63, 342-351 (2011).

44. Friedenstein, A. J., Gorskaja, J. F. \& Kulagina, N. N. Fibroblast precursors in normal and irradiated mouse hematopoietic organs. Exp. Hematol. 4, 267-274 (1976).

45. Liu, Y. et al. Hydrogen sulfide maintains mesenchymal stem cell function and bone homeostasis via regulation of $\mathrm{Ca}(2+)$ channel sulfhydration. Cell Stem Cell 15, 66-78 (2014).

46. Lian, J. B. et al. MicroRNA control of bone formation and homeostasis. Nat. Rev. Endocrinol. 8, 212-227 (2012).

47. Zaidi, M. Skeletal remodeling in health and disease. Nat. Med. 13, 791-801 (2007).

48. Guo, Y. et al. BMP-IHH-mediated interplay between mesenchymal stem cells and osteoclasts supports calvarial bone homeostasis and repair. Bone Res. 6, 30 (2018).

49. Sui, B. et al. Allogeneic mesenchymal stem cell therapy promotes osteoblastogenesis and prevents glucocorticoid-induced osteoporosis. Stem Cells Transl. Med. 5, 1238-1246 (2016). 
50. Shang, F. et al. The effect of licochalcone A on cell-aggregates ECM secretion and osteogenic differentiation during bone formation in metaphyseal defects in ovariectomized rats. Biomaterials 35, 2789-2797 (2014).

51. Sui, B. D. et al. Gender-independent efficacy of mesenchymal stem cell therapy in sex hormone-deficient bone loss via immunosuppression and resident stem cell recovery. Exp. Mol. Med. 50, 166 (2018).

52. Liu, Y. et al. Integration of a calcined bovine bone and BMSC-sheet 3D scaffold and the promotion of bone regeneration in large defects. Biomaterials 34, 9998-10006 (2013).

53. Zuk, P. A. et al. Multilineage cells from human adipose tissue: implications for cell-based therapies. Tissue Eng. 7, 211-228 (2001).

54. Chen, F. M., Sun, H. H., Lu, H. \& Yu, Q. Stem cell-delivery therapeutics for periodontal tissue regeneration. Biomaterials 33, 6320-6344 (2012).

55. Boeloni, J. N., Ocarino, N. M., Goes, A. M. \& Serakides, R. Comparative study of osteogenic differentiation potential of mesenchymal stem cells derived from bone marrow and adipose tissue of osteoporotic female rats. Connect Tissue Res. 55, 103-114 (2014)

56. Beane, O. S., Fonseca, V. C., Cooper, L. L., Koren, G. \& Darling, E. M. Impact of aging on the regenerative properties of bone marrow-, muscle-, and adiposederived mesenchymal stem/stromal cells. PLoS ONE 9, e115963 (2014).

57. Chen, H. T. et al. Proliferation and differentiation potential of human adiposederived mesenchymal stem cells isolated from elderly patients with osteoporotic fractures. J. Cell. Mol. Med. 16, 582-593 (2012).

58. Mirsaidi, A. et al. Telomere length, telomerase activity and osteogenic differentiation are maintained in adipose-derived stromal cells from senile osteoporotic SAMP6 mice. J. Tissue Eng. Regen. Med. 6, 378-390 (2012)

59. Zheng, C. X. et al. Adipose mesenchymal stem cells from osteoporotic donors preserve functionality and modulate systemic inflammatory microenvironment in osteoporotic cytotherapy. Sci. Rep. 8, 5215 (2018).

60. Ye, X. et al. Adipose-derived stem cells alleviate osteoporosis by enhancing osteogenesis and inhibiting adipogenesis in a rabbit model. Cytotherapy 16, 1643-1655 (2014).

61. Pei, M. et al. A comparison of tissue engineering based repair of calvarial defects using adipose stem cells from normal and osteoporotic rats. Bone 78, 1-10 (2015).

62. Lee, K. et al. Systemic transplantation of human adipose-derived stem cells stimulates bone repair by promoting osteoblast and osteoclast function. J. Cell. Mol. Med. 15, 2082-2094 (2011).

63. Mirsaidi, A. et al. Therapeutic potential of adipose-derived stromal cells in agerelated osteoporosis. Biomaterials 35, 7326-7335 (2014).

64. Gronthos, S., Mankani, M., Brahim, J., Robey, P. G. \& Shi, S. Postnatal human dental pulp stem cells (DPSCs) in vitro and in vivo. Proc. Natl Acad. Sci. USA 97, 13625-13630 (2000).

65. Laino, G. et al. A new population of human adult dental pulp stem cells: a useful source of living autologous fibrous bone tissue (LAB). J. Bone Miner. Res. 20, 1394-1402 (2005).

66. Shi, S. \& Gronthos, S. Perivascular niche of postnatal mesenchymal stem cells in human bone marrow and dental pulp. J. Bone Miner. Res. 18, 696-704 (2003).

67. Gronthos, S. et al. Stem cell properties of human dental pulp stem cells. J. Dent. Res. 81, 531-535 (2002)

68. Arthur, A., Rychkov, G., Shi, S., Koblar, S. A. \& Gronthos, S. Adult human dental pulp stem cells differentiate toward functionally active neurons under appropriate environmental cues. Stem Cells 26, 1787-1795 (2008).

69. Ratajczak, J. et al. The neurovascular properties of dental stem cells and their importance in dental tissue engineering. Stem Cells Int. 2016, 9762871 (2016).

70. Zhao, $\mathrm{H}$. et al. Secretion of shh by a neurovascular bundle niche supports mesenchymal stem cell homeostasis in the adult mouse incisor. Cell Stem Cell 14, 160-173 (2014)

71. Xuan, K. et al. Deciduous autologous tooth stem cells regenerate dental pulp after implantation into injured teeth. Sci. Transl. Med. 10, eaaf3227 (2018).

72. Kuang, R. et al. Nanofibrous spongy microspheres for the delivery of hypoxiaprimed human dental pulp stem cells to regenerate vascularized dental pulp. Acta Biomater. 33, 225-234 (2016).

73. lohara, K. et al. A novel combinatorial therapy with pulp stem cells and granulocyte colony-stimulating factor for total pulp regeneration. Stem Cells Transl. Med. 2, 521-533 (2013)

74. Seo, B. M. et al. Investigation of multipotent postnatal stem cells from human periodontal ligament. Lancet 364, 149-155 (2004).

75. Ding, G. et al. Allogeneic periodontal ligament stem cell therapy for periodontitis in swine. Stem Cells 28, 1829-1838 (2010).

76. Liu, Y. et al. Periodontal ligament stem cell-mediated treatment for periodontitis in miniature swine. Stem Cells 26, 1065-1073 (2008).

77. Silverio, K. G. et al. Mesenchymal stem cell properties of periodontal ligament cells from deciduous and permanent teeth. J. Periodontol. 81, 1207-1215 (2010).

78. Seo, B. M. et al. Recovery of stem cells from cryopreserved periodontal ligament. J. Dent. Res. 84, 907-912 (2005).
79. Miura, M. et al. SHED: stem cells from human exfoliated deciduous teeth. Proc. Natl Acad. Sci. USA 100, 5807-5812 (2003).

80. Cordeiro, M. M. et al. Dental pulp tissue engineering with stem cells from exfoliated deciduous teeth. J. Endod. 34, 962-969 (2008).

81. Shi, S. et al. The efficacy of mesenchymal stem cells to regenerate and repair dental structures. Orthod. Craniofac. Res. 8, 191-199 (2005).

82. Kunimatsu, R. et al. Comparative characterization of stem cells from human exfoliated deciduous teeth, dental pulp, and bone marrow-derived mesenchymal stem cells. Biochem. Biophys. Res. Commun. 501, 193-198 (2018).

83. Nakamura, S. et al. Stem cell proliferation pathways comparison between human exfoliated deciduous teeth and dental pulp stem cells by gene expression profile from promising dental pulp. J. Endod. 35, 1536-1542 (2009).

84. Wang, J. et al. Stem cells from human-exfoliated deciduous teeth can differentiate into dopaminergic neuron-like cells. Stem Cells Dev. 19, 1375-1383 (2010).

85. Ma, L. et al. Cryopreserved dental pulp tissues of exfoliated deciduous teeth is a feasible stem cell resource for regenerative medicine. PLOS ONE 7, e51777 (2012).

86. Sonoyama, W. et al. Characterization of the apical papilla and its residing stem cells from human immature permanent teeth: a pilot study. J. Endod. 34, 166-171 (2008).

87. Huang, G. T. et al. The hidden treasure in apical papilla: the potential role in pulp/dentin regeneration and bioroot engineering. J. Endod. 34, 645-651 (2008).

88. Sonoyama, W. et al. Mesenchymal stem cell-mediated functional tooth regeneration in swine. PLoS ONE 1, e79 (2006).

89. Bakopoulou, A. et al. Comparative analysis of in vitro osteo/odontogenic differentiation potential of human dental pulp stem cells (DPSCs) and stem cells from the apical papilla (SCAP). Arch. Oral. Biol. 56, 709-721 (2011).

90. Han, C. et al. Periapical follicle stem cell: a promising candidate for cementum/ periodontal ligament regeneration and bio-root engineering. Stem Cells Dev. 19, 1405-1415 (2010).

91. Liao, J., Al Shahrani, M., Al-Habib, M., Tanaka, T. \& Huang, G. T. Cells isolated from inflamed periapical tissue express mesenchymal stem cell markers and are highly osteogenic. J. Endod. 37, 1217-1224 (2011).

92. Luan, X., Ito, Y., Dangaria, S. \& Diekwisch, T. G. Dental follicle progenitor cell heterogeneity in the developing mouse periodontium. Stem Cells Dev. 15, 595-608 (2006).

93. Handa, K. et al. Progenitor cells from dental follicle are able to form cementum matrix in vivo. Connect Tissue Res. 43, 406-408 (2002).

94. Yao, S., Pan, F., Prpic, V. \& Wise, G. E. Differentiation of stem cells in the dental follicle. J. Dent. Res. 87, 767-771 (2008).

95. Kemoun, P. et al. Human dental follicle cells acquire cementoblast features under stimulation by BMP-2/-7 and enamel matrix derivatives (EMD) in vitro. Cell Tissue Res. 329, 283-294 (2007).

96. Yokoi, T. et al. Establishment of immortalized dental follicle cells for generating periodontal ligament in vivo. Cell Tissue Res. 327, 301-311 (2007).

97. Bai, Y. et al. Cementum- and periodontal ligament-like tissue formation by dental follicle cell sheets co-cultured with Hertwig's epithelial root sheath cells. Bone 48, 1417-1426 (2011).

98. Guo, W. et al. Heterogeneous dental follicle cells and the regeneration of complex periodontal tissues. Tissue Eng. Part A 18, 459-470 (2012).

99. Neves, J., Sousa-Victor, P. \& Jasper, H. Rejuvenating strategies for stem cellbased therapies in aging. Cell Stem Cell 20, 161-175 (2017).

100. Pacelli, S. et al. Strategies to develop endogenous stem cell-recruiting bioactive materials for tissue repair and regeneration. Adv. Drug Deliv. Rev. 120, 50-70 (2017).

101. Xia, H. M. et al. Tissue repair and regeneration with endogenous stem cells. Nat. Rev. Mater. 3, 174-193 (2018)

102. Xue, P. et al. Decreased MORF leads to prolonged endoplasmic reticulum stress in periodontitis-associated chronic inflammation. Cell Death Differ. 23, 1862-1872 (2016).

103. Liu, Y. et al. Chronic high dose alcohol induces osteopenia via activation of mTOR signaling in bone marrow mesenchymal stem cells. Stem Cells 34, 2157-2168 (2016).

104. Chen, C. et al. mTOR inhibition rescues osteopenia in mice with systemic sclerosis. J. Exp. Med. 212, 73-91 (2015)

105. Li, C. et al. Lipopolysaccharide differentially affects the osteogenic differentiation of periodontal ligament stem cells and bone marrow mesenchymal stem cells through toll-like receptor 4 mediated nuclear factor kappaB pathway. Stem Cell Res. Ther. 5, 67 (2014).

106. Liu, N. et al. High levels of beta-catenin signaling reduce osteogenic differentiation of stem cells in inflammatory microenvironments through inhibition of the noncanonical Wnt pathway. J. Bone Miner. Res. 26, 2082-2095 (2011).

107. Li, J. et al. BMl-1 mediates estrogen-deficiency-induced bone loss by inhibiting reactive oxygen species accumulation and T cell activation. J. Bone Miner. Res. 32, 962-973 (2017) 
108. Lean, J. M. et al. A crucial role for thiol antioxidants in estrogen-deficiency bone loss. J. Clin. Investig. 112, 915-923 (2003).

109. Kim, J. H., Singhal, V., Biswal, S., Thimmulappa, R. K. \& DiGirolamo, D. J. Nrf2 is required for normal postnatal bone acquisition in mice. Bone Res. 2, 14033 (2014).

110. Lv, Y. J. et al. Resveratrol counteracts bone loss via mitofilin-mediated osteogenic improvement of mesenchymal stem cells in senescence-accelerated mice. Theranostics 8, 2387-2406 (2018).

111. Bhattarai, G., Poudel, S. B., Kook, S. H. \& Lee, J. C. Resveratrol prevents alveolar bone loss in an experimental rat model of periodontitis. Acta Biomater. 29, 398-408 (2016).

112. Kara, A. et al. Immune modulatory and antioxidant effects of melatonin in experimental periodontitis in rats. Free Radic. Biol. Med. 55, 21-26 (2013).

113. Jing, $\mathrm{H}$. et al. Suppression of EZH2 prevents the shift of osteoporotic MSC fate to adipocyte and enhances bone formation during osteoporosis. Mol. Ther. 24, 217-229 (2016).

114. Lv, L. et al. Lysine-specific demethylase 1 inhibitor rescues the osteogenic ability of mesenchymal stem cells under osteoporotic conditions by modulating H3K4 methylation. Bone Res. 4, 16037 (2016).

115. Wang, C. et al. KDM5A controls bone morphogenic protein 2-induced osteogenic differentiation of bone mesenchymal stem cells during osteoporosis. Cell Death Dis. 7, e2335 (2016)

116. Zhang, X., Li, Y., Chen, Y. E., Chen, J. \& Ma, P. X. Cell-free 3D scaffold with twostage delivery of miRNA-26a to regenerate critical-sized bone defects. Nat. Commun. 7, 10376 (2016).

117. Yoshizuka, M. et al. Inhibition of microRNA-222 expression accelerates bone healing with enhancement of osteogenesis, chondrogenesis, and angiogenesis in a rat refractory fracture model. J. Orthop. Sci. 21, 852-858 (2016).

118. Li, C. J. et al. MicroRNA-188 regulates age-related switch between osteoblast and adipocyte differentiation. J. Clin. Investig. 125, 1509-1522 (2015).

119. Li, Y. et al. MiR-26a rescues bone regeneration deficiency of mesenchymal stem cells derived from osteoporotic mice. Mol. Ther. 23, 1349-1357 (2015).

120. Khaldoyanidi, S. Directing stem cell homing. Cell Stem Cell 2, 198-200 (2008).

121. Laird, D. J., von Andrian, U. H. \& Wagers, A. J. Stem cell trafficking in tissue development, growth, and disease. Cell 132, 612-630 (2008).

122. Eramo, S., Natali, A., Pinna, R. \& Milia, E. Dental pulp regeneration via cell homing. Int. Endod. J. 51, 405-419 (2018).

123. Guan, M. et al. Directing mesenchymal stem cells to bone to augment bone formation and increase bone mass. Nat. Med. 18, 456-462 (2012).

124. Chen, P. et al. Radially oriented collagen scaffold with SDF-1 promotes osteochondral repair by facilitating cell homing. Biomaterials 39, 114-123 (2015).

125. Cai, X. et al. Periodontal regeneration via chemoattractive constructs. J. Clin. Periodontol. 45, 851-860 (2018).

126. Lee, C. H. et al. Regeneration of the articular surface of the rabbit synovial joint by cell homing: a proof of concept study. Lancet 376, 440-448 (2010).

127. Ogawa, K. et al. Periodontal tissue engineering by nano beta-tricalcium phosphate scaffold and fibroblast growth factor-2 in one-wall infrabony defects of dogs. J. Periodontal Res. 51, 758-767 (2016).

128. Zhang, X. S. et al. Local ex vivo gene therapy with bone marrow stromal cells expressing human BMP4 promotes endosteal bone formation in mice. J. Gene. Med. 6, 4-15 (2004).

129. Devine, S. M. et al. Mesenchymal stem cells are capable of homing to the bone marrow of non-human primates following systemic infusion. Exp. Hematol. 29, 244-255 (2001).

130. Huang, S., Xu, L., Zhang, Y., Sun, Y. \& Li, G. Systemic and local administration of allogeneic bone marrow-derived mesenchymal stem cells promotes fracture healing in rats. Cell Transplant. 24, 2643-2655 (2015).

131. Lien, C. Y., Ho, K. C.-Y., Lee, O. K., Blunn, G. W. \& Su, Y. Restoration of bone mass and strength in glucocorticoid-treated mice by systemic transplantation of CXCR4 and cbfa-1 co-expressing mesenchymal stem cells. J. Bone Miner. Res. 24 837-848 (2009).

132. Yao, W. et al. Reversing bone loss by directing mesenchymal stem cells to bone. Stem Cells 31, 2003-2014 (2013).

133. Cho, S. W. et al. Transplantation of mesenchymal stem cells overexpressing RANK-FC or CXCR4 prevents bone loss in ovariectomized mice. Mol. Ther. 17, 1979-1987 (2009).

134. Li, Y. S. et al. Allogeneic mesenchymal stem cell therapy for bisphosphonaterelated jaw osteonecrosis in swine. Stem Cells Dev. 22, 2047-2056 (2013).

135. Liu, Y., Yang, R. L. \& Shi, S. T. Systemic infusion of mesenchymal stem cells improves cell-based bone regeneration via upregulation of regulatory $\mathrm{T}$ cells. Tissue Eng. Part A 21, 498-509 (2015).

136. Ma, L. et al. Transplantation of mesenchymal stem cells ameliorates secondary osteoporosis through interleukin-17-impaired functions of recipient bone marrow mesenchymal stem cells in MRL/lpr mice. Stem Cell Res. Ther. 6, 104 (2015).
137. Kikuiri, T. et al. Cell-based immunotherapy with mesenchymal stem cells cures bisphosphonate-related osteonecrosis of the jaw-like disease in mice. J. Bone Miner. Res. 25, 1668-1679 (2010).

138. Yang, J. et al. Cell delivery in regenerative medicine: the cell sheet engineering approach. J. Control Release 116, 193-203 (2006).

139. Ahrens, C. C., Dong, Z. \& Li, W. Engineering cell aggregates through incorporated polymeric microparticles. Acta Biomater. 62, 64-81 (2017).

140. Yang, J. et al. Reconstruction of functional tissues with cell sheet engineering. Biomaterials 28, 5033-5043 (2007).

141. Zheng, C. X. et al. Reconstruction of structure and function in tissue engineering of solid organs: toward simulation of natural development based on decellularization. J. Tissue Eng. Regen. Med. 12, 1432-1447 (2018).

142. Kelm, J. M. \& Fussenegger, M. Scaffold-free cell delivery for use in regenerative medicine. Adv. Drug Deliv. Rev. 62, 753-764 (2010).

143. Yan, J. et al. Non-viral oligonucleotide antimiR-138 delivery to mesenchymal stem cell sheets and the effect on osteogenesis. Biomaterials 35, 7734-7749 (2014).

144. Jiang, Z. et al. Light-controlled BMSC sheet-implant complexes with improved osteogenesis via an LRP5/beta-catenin/Runx2 regulatory loop. ACS Appl. Mater. Interfaces 9, 34674-34686 (2017).

145. Flores, M. G. et al. Cementum-periodontal ligament complex regeneration using the cell sheet technique. J. Periodontal Res. 43, 364-371 (2008).

146. Tsumanuma, Y. et al. Comparison of different tissue-derived stem cell sheets for periodontal regeneration in a canine 1-wall defect model. Biomaterials 32, 5819-5825 (2011)

147. Zhou, Y., Li, Y., Mao, L. \& Peng, H. Periodontal healing by periodontal ligament cell sheets in a teeth replantation model. Arch. Oral. Biol. 57, 169-176 (2012).

148. Flores, M. G. et al. Periodontal ligament cell sheet promotes periodontal regeneration in athymic rats. J. Clin. Periodontol. 35, 1066-1072 (2008).

149. Iwata, T. et al. Periodontal regeneration with multi-layered periodontal ligament-derived cell sheets in a canine model. Biomaterials 30, 2716-2723 (2009).

150. Iwata, T. et al. Periodontal regeneration with autologous periodontal ligamentderived cell sheets - a safety and efficacy study in ten patients. Regen. Ther. $\mathbf{9}$, 38-44 (2018).

151. Feng, F. et al. Utility of PDL progenitors for in vivo tissue regeneration: a report of 3 cases. Oral. Dis. 16, 20-28 (2010).

152. Wang, Y. J. et al. Resveratrol enhances the functionality and improves the regeneration of mesenchymal stem cell aggregates. Exp. Mol. Med. 50, 74 (2018).

153. Guo, W. et al. Scaffold-free cell pellet transplantations can be applied to periodontal regeneration. Cell Transplant. 23, 181-194 (2014).

154. Zhu, B. et al. Tissue-specific composite cell aggregates drive periodontium tissue regeneration by reconstructing a regenerative microenvironment. J. Tissue Eng. Regen. Med. 11, 1792-1805 (2017).

155. Zhou, Y. et al. Combined marrow stromal cell-sheet techniques and highstrength biodegradable composite scaffolds for engineered functional bone grafts. Biomaterials 28, 814-824 (2007).

156. Dan, $\mathrm{H}$. et al. The influence of cellular source on periodontal regeneration using calcium phosphate coated polycaprolactone scaffold supported cell sheets. Biomaterials 35, 113-122 (2014).

157. Raisz, L. G. Pathogenesis of osteoporosis: concepts, conflicts, and prospects. J. Clin. Investig. 115, 3318-3325 (2005).

158. Almeida, M., Han, L., Martin-Millan, M., O'Brien, C. A. \& Manolagas, S. C. Oxidative stress antagonizes Wnt signaling in osteoblast precursors by diverting betacatenin from $\mathrm{T}$ cell factor- to forkhead box O-mediated transcription. J. Biol. Chem. 282, 27298-27305 (2007).

159. Joswig, A. J. et al. Repeated intra-articular injection of allogeneic mesenchymal stem cells causes an adverse response compared to autologous cells in the equine model. Stem Cell Res. Ther. 8, 42 (2017).

160. Liao, L. et al. TNF-alpha inhibits FoxO1 by upregulating miR-705 to aggravate oxidative damage in bone marrow-derived mesenchymal stem cells during osteoporosis. Stem Cells 34, 1054-1067 (2016).

161. Liao, L. et al. Redundant miR-3077-5p and miR-705 mediate the shift of mesenchymal stem cell lineage commitment to adipocyte in osteoporosis bone marrow. Cell Death Dis. 4, e600 (2013).

162. Leskela, H. V. et al. Estrogen receptor alpha genotype confers interindividual variability of response to estrogen and testosterone in mesenchymal-stem-cellderived osteoblasts. Bone 39, 1026-1034 (2006).

163. Almeida, M. et al. Estrogens and androgens in skeletal physiology and pathophysiology. Physiol. Rev. 97, 135-187 (2017).

164. Almeida, M. et al. Skeletal involution by age-associated oxidative stress and its acceleration by loss of sex steroids. J. Biol. Chem. 282, 27285-27297 (2007).

165. Jing, $\mathrm{H}$. et al. Epigenetic inhibition of Wnt pathway suppresses osteogenic differentiation of BMSCs during osteoporosis. Cell Death Dis. 9, 176 (2018). 
166. Yang, N. et al. Tumor necrosis factor alpha suppresses the mesenchymal stem cell osteogenesis promoter miR-21 in estrogen deficiency-induced osteoporosis. J. Bone Miner. Res. 28, 559-573 (2013).

167. Li, J. et al. Dexamethasone shifts bone marrow stromal cells from osteoblasts to adipocytes by C/EBPalpha promoter methylation. Cell Death Dis. 4, e832 (2013).

168. Wei, P., Dove, K. K., Bensard, C., Schell, J. C. \& Rutter, J. The force is strong with this one: Metabolism (over)powers stem cell fate. Trends Cell Biol. 28, 551-559 (2018).

169. Khacho, M. \& Slack, R. S. Mitochondrial activity in the regulation of stem cell selfrenewal and differentiation. Curr. Opin. Cell Biol. 49, 1-8 (2017).

170. Riddle, R. C. \& Clemens, T. L. Bone cell bioenergetics and skeletal energy homeostasis. Physiol. Rev. 97, 667-698 (2017).

171. Shum, L. C., White, N. S., Mills, B. N., Bentley, K. L. \& Eliseev, R. A. Energy metabolism in mesenchymal stem cells during osteogenic differentiation. Stem Cells Dev. 25, 114-122 (2016).

172. Lo, T., Ho, J. H., Yang, M. H. \& Lee, O. K. Glucose reduction prevents replicative senescence and increases mitochondrial respiration in human mesenchymal stem cells. Cell Transplant. 20, 813-825 (2011).

173. Guan, C. C. et al. Sonic hedgehog alleviates the inhibitory effects of high glucose on the osteoblastic differentiation of bone marrow stromal cells. Bone $\mathbf{4 5}$, 1146-1152 (2009).

174. Napoli, N. et al. Mechanisms of diabetes mellitus-induced bone fragility. Nat. Rev. Endocrinol. 13, 208-219 (2017).

175. Kume, S. et al. Advanced glycation end-products attenuate human mesenchymal stem cells and prevent cognate differentiation into adipose tissue, cartilage, and bone. J. Bone Miner. Res. 20, 1647-1658 (2005).

176. Li, J., He, W., Liao, B. \& Yang, J. FFA-ROS-P53-mediated mitochondrial apoptosis contributes to reduction of osteoblastogenesis and bone mass in type 2 diabetes mellitus. Sci. Rep. 5, 12724 (2015)

177. Lalla, E. \& Papapanou, P. N. Diabetes mellitus and periodontitis: a tale of two common interrelated diseases. Nat. Rev. Endocrinol. 7, 738-748 (2011).

178. Lalla, E. et al. Blockade of RAGE suppresses periodontitis-associated bone loss in diabetic mice. J. Clin. Investig. 105, 1117-1124 (2000).

179. Liu, Q. et al. DKK1 rescues osteogenic differentiation of mesenchymal stem cells isolated from periodontal ligaments of patients with diabetes mellitus induced periodontitis. Sci. Rep. 5, 13142 (2015).

180. Giannopoulou, C, Krause, K. H. \& Muller, F. The NADPH oxidase NOX2 plays a role in periodontal pathologies. Semin. Immunopathol. 30, 273-278 (2008).

181. Abdelmagid, S. M., Barbe, M. F. \& Safadi, F. F. Role of inflammation in the aging bones. Life Sci. 123, 25-34 (2015).

182. Wang, L. et al. IFN-gamma and TNF-alpha synergistically induce mesenchymal stem cell impairment and tumorigenesis via NFkappaB signaling. Stem Cells 31, 1383-1395 (2013).

183. Li, B. et al. GCN5 modulates osteogenic differentiation of periodontal ligament stem cells through DKK1 acetylation in inflammatory microenvironment. Sci. Rep. 6, 26542 (2016).

184. Liu, Y. et al. MiR-17 modulates osteogenic differentiation through a coherent feed-forward loop in mesenchymal stem cells isolated from periodontal ligaments of patients with periodontitis. Stem Cells 29, 1804-1816 (2011).

185. Yang, N. et al. Tumor necrosis factor-alpha suppresses adipogenic and osteogenic differentiation of human periodontal ligament stem cell by inhibiting miR21/Spry1 functional axis. Differentiation 97, 33-43 (2017)

186. Yang, $H$. et al. Comparison of mesenchymal stem cells derived from gingival tissue and periodontal ligament in different incubation conditions. Biomaterials 34, 7033-7047 (2013).

187. Zhu, M. et al. The effect of age on osteogenic, adipogenic and proliferative potential of female adipose-derived stem cells. J. Tissue Eng. Regen. Med. 3, 290-301 (2009).

188. Veronesi, F., Pagani, S., Bella, E. D., Giavaresi, G. \& Fini, M. Estrogen deficiency does not decrease the in vitro osteogenic potential of rat adipose-derived mesenchymal stem cells. Age 36, 9647 (2014).

189. Galleu, A. et al. Apoptosis in mesenchymal stromal cells induces in vivo recipient-mediated immunomodulation. Sci. Transl. Med. 9, eaam7828 (2017).

190. Papadopoulou, A. et al. Mesenchymal stem cells are conditionally therapeutic in preclinical models of rheumatoid arthritis. Ann. Rheum. Dis. 71, 1733-1740 (2012)

191. te Velde, E. R. \& van Leusden, H. A. Hormonal treatment for the climacteric: alleviation of symptoms and prevention of postmenopausal disease. Lancet $\mathbf{3 4 3}$, 654-658 (1994)

192. Shao, B. et al. Estrogen preserves Fas ligand levels by inhibiting microRNA-181a in bone marrow-derived mesenchymal stem cells to maintain bone remodeling balance. FASEB J. 29, 3935-3944 (2015).

193. Speakman, J. R. \& Mitchell, S. E. Caloric restriction. Mol. Asp. Med. 32, 159-221 (2011).
194. Villareal, D. T. et al. Effect of two-year caloric restriction on bone metabolism and bone mineral density in non-obese younger adults: a randomized clinical trial. J. Bone Miner. Res. 31, 40-51 (2016).

195. Behrendt, A. K. et al. Dietary restriction-induced alterations in bone phenotype: effects of lifelong versus short-term caloric restriction on femoral and vertebral bone in C57BL/6 mice. J. Bone Miner. Res. 31, 852-863 (2016).

196. Pscherer, S., Kostev, K., Dippel, F. W. \& Rathmann, W. Fracture risk in patients with type 2 diabetes under different antidiabetic treatment regimens: a retrospective database analysis in primary care. Diabetes Metab. Syndr. Obes. 9, 17-23 (2016).

197. Campos Pastor, M. M., Lopez-lbarra, P. J., Escobar-Jimenez, F., Serrano Pardo, M. D. \& Garcia-Cervigon, A. G. Intensive insulin therapy and bone mineral density in type 1 diabetes mellitus: a prospective study. Osteoporos. Int. 11, 455-459 (2000).

198. Pearson, K. J. et al. Resveratrol delays age-related deterioration and mimics transcriptional aspects of dietary restriction without extending life span. Cell Metab. 8, 157-168 (2008)

199. Molinuevo, M. S. et al. Effect of metformin on bone marrow progenitor cell differentiation: in vivo and in vitro studies. J. Bone Miner. Res. 25, 211-221 (2010).

200. Liu, W. et al. Alpl prevents bone ageing sensitivity by specifically regulating senescence and differentiation in mesenchymal stem cells. Bone Res. 6, 27 (2018).

201. Zhang, K. et al. Preservation of high-fat diet-induced femoral trabecular bone loss through genetic target of TNF-alpha. Endocrine 50, 239-249 (2015).

202. Yamaza, T. et al. Pharmacologic stem cell based intervention as a new approach to osteoporosis treatment in rodents. PLOS ONE 3, e2615 (2008).

203. Liu, Y. et al. Acetylsalicylic acid treatment improves differentiation and immunomodulation of SHED. J. Dent. Res. 94, 209-218 (2015).

204. Shuai, Y. et al. Melatonin treatment improves mesenchymal stem cells therapy by preserving stemness during long-term in vitro expansion. Theranostics $\mathbf{6}$, 1899-1917 (2016).

205. Sonoda, S. et al. Interferon-gamma improves impaired dentinogenic and immunosuppressive functions of irreversible pulpitis-derived human dental pulp stem cells. Sci. Rep. 6, 19286 (2016).

206. Duque, G. et al. Interferon-gamma plays a role in bone formation in vivo and rescues osteoporosis in ovariectomized mice. J. Bone Miner. Res. 26, 1472-1483 (2011).

207. Hao, H. et al. Multiple intravenous infusions of bone marrow mesenchymal stem cells reverse hyperglycemia in experimental type 2 diabetes rats. Biochem. Biophys. Res. Commun. 436, 418-423 (2013).

208. Ho, J. H. et al. Multiple intravenous transplantations of mesenchymal stem cells effectively restore long-term blood glucose homeostasis by hepatic engraftment and beta-cell differentiation in streptozocin-induced diabetic mice. Cell Transplant. 21, 997-1009 (2012).

209. An, Y. et al. Bone marrow mesenchymal stem cell aggregate: an optimal cell therapy for full-layer cutaneous wound vascularization and regeneration. Sci. Rep. 5, 17036 (2015).

210. Lin, K. et al. Enhanced osteoporotic bone regeneration by strontium-substituted calcium silicate bioactive ceramics. Biomaterials 34, 10028-10042 (2013).

211. Xia, L. et al. Akermanite bioceramics promote osteogenesis, angiogenesis and suppress osteoclastogenesis for osteoporotic bone regeneration. Sci. Rep. 6, 22005 (2016).

212. Zhang, Y., Cheng, N., Miron, R., Shi, B. \& Cheng, X. Delivery of PDGF-B and BMP-7 by mesoporous bioglass/silk fibrin scaffolds for the repair of osteoporotic defects. Biomaterials 33, 6698-6708 (2012).

213. $\mathrm{Wu}, \mathrm{Y}$. et al. Evaluation of osteogenesis and angiogenesis of icariin in local controlled release and systemic delivery for calvarial defect in ovariectomized rats. Sci. Rep. 7, 5077 (2017).

214. Ranganath, S. H., Levy, O., Inamdar, M. S. \& Karp, J. M. Harnessing the mesenchymal stem cell secretome for the treatment of cardiovascular disease. Cell Stem Cell 10, 244-258 (2012)

215. Rani, S., Ryan, A. E., Griffin, M. D. \& Ritter, T. Mesenchymal stem cell-derived extracellular vesicles: toward cell-free therapeutic applications. Mol. Ther. 23, 812-823 (2015).

216. Riazifar, M., Pone, E. J., Lotvall, J. \& Zhao, W. Stem cell extracellular vesicles: Extended messages of regeneration. Annu. Rev. Pharmacol. Toxicol. 57, 125-154 (2017)

217. Tao, S. C. et al. Exosomes derived from miR-140-5p-overexpressing human synovial mesenchymal stem cells enhance cartilage tissue regeneration and prevent osteoarthritis of the knee in a rat model. Theranostics 7, 180-195 (2017).

218. Liu, X. et al. Exosomes secreted from human-induced pluripotent stem cellderived mesenchymal stem cells prevent osteonecrosis of the femoral head by promoting angiogenesis. Int. J. Biol. Sci. 13, 232-244 (2017).

219. Zhang, J. et al. Exosomes/tricalcium phosphate combination scaffolds can enhance bone regeneration by activating the PI3K/Akt signaling pathway. Stem Cell Res. Ther. 7, 136 (2016) 
220. Liu, Y. et al. MSC-derived exosomes promote proliferation and inhibit apoptosis of chondrocytes via IncRNA-KLF3-AS1/miR-206/GIT1 axis in osteoarthritis. Cell Cycle 17, 2411-2422 (2018).

221. Li, W. et al. Tissue-engineered bone immobilized with human adipose stem cells-derived exosomes promotes bone regeneration. ACS Appl. Mater. Interfaces 10, 5240-5254 (2018).

222. Qi, X. et al. Exosomes secreted by human-induced pluripotent stem cell-derived mesenchymal stem cells repair critical-sized bone defects through enhanced angiogenesis and osteogenesis in osteoporotic rats. Int. J. Biol. Sci. 12, 836-849 (2016).

223. Pers, Y. M. et al. Adipose mesenchymal stromal cell-based therapy for severe osteoarthritis of the knee: a phase i dose-escalation trial. Stem Cells Transl. Med. 5, 847-856 (2016)

224. Zhao, D. et al. Treatment of early stage osteonecrosis of the femoral head with autologous implantation of bone marrow-derived and cultured mesenchymal stem cells. Bone 50, 325-330 (2012).

225. Tabatabaee, R. M., Saberi, S., Parvizi, J., Mortazavi, S. M. \& Farzan, M. Combining concentrated autologous bone marrow stem cells injection with core decompression improves outcome for patients with early-stage osteonecrosis of the femoral head: a comparative study. J. Arthroplast. 30, 11-15 (2015).

226. Daltro, G. C. et al. Efficacy of autologous stem cell-based therapy for osteonecrosis of the femoral head in sickle cell disease: a five-year follow-up study. Stem Cell Res. Ther. 6, 110 (2015).

227. Mao, Q. et al. The efficacy of targeted intraarterial delivery of concentrated autologous bone marrow containing mononuclear cells in the treatment of osteonecrosis of the femoral head: a five year follow-up study. Bone 57, 509-516 (2013).

228. Thesleff, T. et al. Cranioplasty with adipose-derived stem cells and biomaterial: a novel method for cranial reconstruction. Neurosurgery 68, 1535-1540 (2011).

229. Gjerde, C. et al. Cell therapy induced regeneration of severely atrophied mandibular bone in a clinical trial. Stem Cell Res. Ther. 9, 213 (2018).

230. Chen, F. M. et al. Treatment of periodontal intrabony defects using autologous periodontal ligament stem cells: a randomized clinical trial. Stem Cell Res. Ther. 7, 33 (2016).
231. Xia, L. et al. Effect of nano-structured bioceramic surface on osteogenic differentiation of adipose derived stem cells. Biomaterials 35, 8514-8527 (2014).

232. Li, Y., Xiao, Y. \& Liu, C. The horizon of materiobiology: a perspective on materialguided cell behaviors and tissue engineering. Chem. Rev. 117, 4376-4421 (2017).

233. Liu, Z., Tang, M., Zhao, J., Chai, R. \& Kang, J. Looking into the future: toward advanced $3 \mathrm{D}$ biomaterials for stem-cell-based regenerative medicine. Adv. Mater. 30, e1705388 (2018).

234. Liang, C. et al. Aptamer-functionalized lipid nanoparticles targeting osteoblasts as a novel RNA interference-based bone anabolic strategy. Nat. Med. 21, 288-294 (2015).

235. Zhang, G. et al. A delivery system targeting bone formation surfaces to facilitate RNAi-based anabolic therapy. Nat. Med. 18, 307-314 (2012).

236. Liu, D. et al. Circulating apoptotic bodies maintain mesenchymal stem cell homeostasis and ameliorate osteopenia via transferring multiple cellular factors. Cell Res. 28, 918-933 (2018).

(i) Open Access This article is licensed under a Creative Commons Attribution 4.0 International License, which permits use, sharing, adaptation, distribution and reproduction in any medium or format, as long as you give appropriate credit to the original author(s) and the source, provide a link to the Creative Commons license, and indicate if changes were made. The images or other third party material in this article are included in the article's Creative Commons license, unless indicated otherwise in a credit line to the material. If material is not included in the article's Creative Commons license and your intended use is not permitted by statutory regulation or exceeds the permitted use, you will need to obtain permission directly from the copyright holder. To view a copy of this license, visit http://creativecommons. org/licenses/by/4.0/.

(c) The Author(s) 2019 